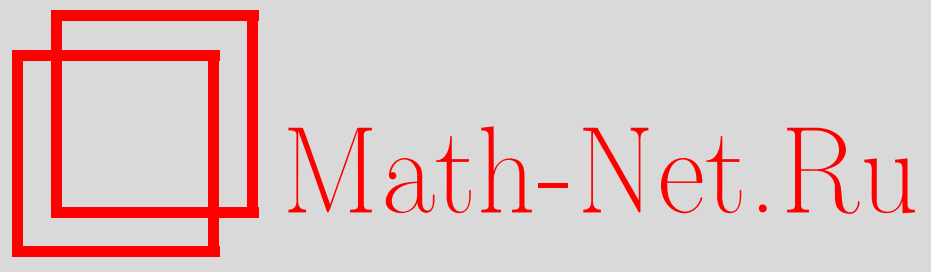

C. С. Платонов, О спектральном синтезе на поэлементно компактных абелевых группах, Матем. сб., 2015, том 206, номер 8, 127-152

DOI: https://doi.org/10.4213/sm8419

Использование Общероссийского математического портала Math-Net.Ru подразумевает, что вы прочитали и согласны с пользовательским соглашением http://www . mathnet.ru/rus/agreement

Параметры загрузки:

IP : 18.208 .226 .222

26 апреля 2023 г., 16:32:37

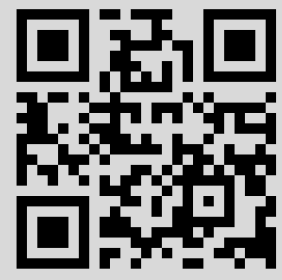




\section{С. С. Платонов \\ О спектральном синтезе на поэлементно компактных абелевых группах}

Пусть $G$ - произвольная локально компактная абелева группа, $C(G)$ пространство всех непрерывных комплекснозначных функций на группе $G$. Замкнутое линейное подпространство $\mathscr{H} \subseteq C(G)$ называется инвариантным подпространством, если оно инвариантно относительно сдвигов $\tau_{y}: f(x) \mapsto f(x y), y \in G$. По определению инвариантное подпространство $\mathscr{H} \subseteq C(G)$ допускает строгий спектральный синтез, если $\mathscr{H}$ совпадает с замыканием в $C(G)$ линейной оболочки всех содержащихся в $\mathscr{H}$ характеров группы $G$. Будем говорить, что на группе $G$ справедлив строгий спектральный синтез в пространстве $C(G)$, если любое инвариантное подпространство $\mathscr{H} \subseteq C(G)$ допускает строгий спектральный синтез. Элемент $x$ топологической группы $G$ называется компактным, если $x$ содержится в некоторой компактной подгруппе группы $G$. Группа $G$ называется поэлементно компактной, если все элементы этой группы компактны. Основным результатом статьи является доказательство того, что на локально компактной абелевой группе $G$ справедлив строгий спектральный синтез в пространстве $C(G)$ тогда и только тогда, когда группа $G$ поэлементно компактная.

Библиография: 14 названий.

Ключевые слова: спектральный синтез, локально компактные абелевы группы, поэлементно компактные группы, преобразование Фурье на группах, функции Брюа-Шварца.

DOI: $10.4213 / \operatorname{sm} 8419$

\section{§ 1. Введение и формулировка основных результатов}

Пусть $G$ - локально компактная абелева группа (LCA-группа), $\mathscr{F}$ - топологическое векторное пространство (ТВП), состоящее из комплекснозначных функций на $G$. Будем называть пространство $\mathscr{F}$ трансляционно инвариантнымм, если $\mathscr{F}$ инвариантно относительно преобразований (сдвигов)

$$
\tau_{y}: f(x) \mapsto f(x y), \quad f(x) \in \mathscr{F}, \quad y \in G,
$$

и все операторы $\tau_{y}$ являются непрерывными операторами в пространстве $\mathscr{F}$.

Замкнутое линейное подпространство $\mathscr{H} \subseteq \mathscr{F}$ называется инвариантным подпространством, если $\tau_{y}(\mathscr{H}) \subseteq \mathscr{H}$ для любого $y \in G$.

Экспоненииальной функцией или обобщенным характером называется произвольный непрерывный гомоморфизм из группы $G$ в мультипликативную

(C) С. С. ПлАтонов, 2015 
группу $\mathbb{C}_{*}:=\mathbb{C} \backslash\{0\}$ ненулевых комплексных чисел. Частным случаем экспоненциальных функций являются характеры - непрерывные гомоморфизмы группы $G$ в группу $\mathbb{T}$ комплексных чисел, по модулю равных 1. Непрерывные гомоморфизмы из группы $G$ в аддитивную группу комплексных чисел называются аддитивными функииями. Функция $x \mapsto P\left(a_{1}(x), \ldots, a_{m}(x)\right)$ на $G$ называется полиномиальной, если $P$ - комплексный полином от $m$ переменных и $a_{1}, \ldots, a_{m}$ - аддитивные функции. Произведение полиномиальной и экспоненциальной функций называется экспоненииальным мономом, а сумма экспоненциальных мономов называется экспоненииальным полиномом на группе $G$.

Пусть $\mathscr{F}$ - трансляционно инвариантное функциональное пространство на группе $G, \mathscr{H}$ - инвариантное подпространство в $\mathscr{F}$.

ОПРедЕЛЕНиЕ 1.1. Инвариантное подпространство $\mathscr{H}$ допускает спектральный синтез, если оно совпадает с замыканием в $\mathscr{F}$ линейной оболочки всех содержащихся в $\mathscr{H}$ экспоненциальных мономов. В пространстве $\mathscr{F}$ справедлив спектральный синтез, если любое инвариантное подпространство $\mathscr{H} \subseteq \mathscr{F}$ допускает спектральный синтез.

Приведенное определение спектрального синтеза берет начало с работы Л. Шварца [1], в которой доказана справедливость спектрального синтеза для случая, когда $G=(\mathbb{R},+)$, а пространство $\mathscr{F}$ может совпадать с пространством $C(\mathbb{R})$ всех непрерывных функций на $\mathbb{R}$ или с пространством $\mathscr{E}(\mathbb{R})=$ $C^{\infty}(\mathbb{R})$ всех бесконечно дифференцируемых функций на $\mathbb{R}$ (если не оговорено противное, то все функции предполагаются комплекснозначными и все классические функциональные пространства рассматриваются с обычными топологиями).

Справедливость или несправедливость спектрального синтеза зависит как от группы $G$, так и от ТВП $\mathscr{F}$. Так, если взять $G=\left(\mathbb{R}^{n},+\right), n \geqslant 2$, то в пространствах $C\left(\mathbb{R}^{n}\right)$ и $\mathscr{E}\left(\mathbb{R}^{n}\right)=C^{\infty}\left(\mathbb{R}^{n}\right)$ спектральный синтез не справедлив (см. [2]), а в пространстве $\mathscr{S}^{\prime}\left(\mathbb{R}^{n}\right)$ обобщенных функций медленного роста на $\mathbb{R}^{n}$ спектральный синтез справедлив (см. [3]). Обзор результатов о спектральном синтезе на LCA-группах см. в [4] и [5]. Одним из наиболее естественных функциональных пространств, где можно изучать спектральный синтез, является пространство $C(G)$ всех непрерывных функций на группе $G$. В общем случае неизвестен критерий, позволяющий определить справедлив или не справедлив спектральный синтез в пространстве $C(G)$. В частном случае, когда группа $G$ дискретная, известно, что спектральный синтез в пространстве $C(G)$ справедлив тогда и только тогда, когда ее ранг без кручения конечен (см. [6]).

Для недискретных LCA-групп большой интерес представляет получение достаточных условий на группу $G$, при которых в пространстве $C(G)$ справедлив спектральный синтез.

Элемент $x$ топологической группы $G$ называется компактным элементом, если $x$ содержится в некоторой компактной подгруппе группы $G$ или, эквивалентно, наименьшая замкнутая подгруппа в $G$, содержащая $x$, компактна. Известно, что если $G$ - LCA-группа, то множество $B$ всех компактных элементов в $G$ образует замкнутую подгруппу в $G$ (см. [7; гл. 2, теорема 9.10]). Будем 
называть топологическую группу $G$ поэлементно компактной, если любой элемент группы $G$ компактен.

Пусть $G$ - поэлементно компактная LCA-группа. Если $\varphi: G \mapsto \mathbb{C}_{*}$ - обобщенный характер группы $G$, то для любого элемента $x \in G$ его образ содержится в некоторой компактной подгруппе группы $\mathbb{C}_{*}$. Но любая компактная подгруппа группы $\mathbb{C}_{*}$ содержится в подгруппе $\mathbb{T}=\{z \in \mathbb{C}:|z|=1\}$, поэтому любой обобщенный характер совпадает с обычным характером. Также легко видеть, что любая аддитивная функция на группе $G$ тождественно равна нулю, поэтому любой экспоненциальный моном на группе $G$ имеет вид $\lambda \chi(x)$, где $\lambda \in \mathbb{C}$, $\chi(x)$ - некоторый характер группы $G$. В связи с этим справедливо следующее предложение.

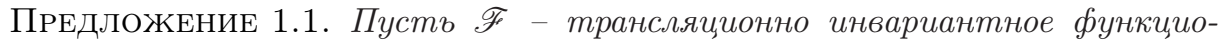
нальное пространство на группе $G, \mathscr{H}$ - инвариантное подпространство в $\mathscr{F}$. Если $G$ - поэлементно компактная LCA-группа, то $\mathscr{H}$ допускает спектральный синтез тогда и толъко тогда, когда $\mathscr{H}$ совпадает с замыканием линейной оболочки всех содержащихся в $\mathscr{H}$ характеров группъ $G$.

Для любой LCA-группы $G$ пусть $\widehat{G}$ - множество всех характеров группы $G$. Снабженное компактно-открытой топологией и операцией поточечного умножения характеров, множество $\widehat{G}$ становится LCA-группой (см. [7]-[9]). Если $\mathscr{H}$ - инвариантное подпространство, то спектром $\mathscr{H}$ будем называть множество

$$
\sigma_{\mathscr{H}}:=\{\chi \in \widehat{G}: \chi \in \mathscr{H}\} .
$$

Если $G$ - поэлементно компактная LCA-группа и инвариантное подпространство $\mathscr{H}$ допускает спектральный синтез, то $\mathscr{H}$ однозначно восстанавливается по своему спектру.

Основным результатом работы является следующая теорема.

Теорема 1.1. Пусть $G$ - произвольная поэлементно компактная LCAгрупnа. Тогда:

1) спектральный синтез справедлив в пространстве $C(G)$ всех непрерывных функиий на группе $G$;

2) для того чтобы подмножество $\sigma \subseteq \widehat{G}$ было спектром некоторого инвариантного подпространства в $C(G)$, необходимо и достаточно, чтобы $\sigma$ было замкнутым подмножеством в $\widehat{G}$.

В частном случае, когда $G$ является нульмерной поэлементно компактной LCA-группой, теорема 1.1 была доказана ранее в [4]. В настоящей работе установлено, что условие нульмерности излишне и тем самым доказана справедливость спектрального синтеза в пространстве $C(G)$ для более широкого класса LCA-групп. Отметим также, что справедливость спектрального синтеза в пространстве $C(G)$ для любой компактной группы $G$ была установлена ранее в [10].

Пусть $G$ - произвольная LCA-группа, $\mathscr{F}$ - трансляционно инвариантное функциональное пространство на группе $G, \mathscr{H}$ - инвариантное подпространство в $\mathscr{F}$. Будем говорить, что инвариантное подпространство $\mathscr{H}$ допускает 
строгий спектралъный синтез, если оно совпадает с замыканием линейной оболочки всех содержащихся в $\mathscr{H}$ характеров группы $G$. B пространстве $\mathscr{F}$ справедлив строгий спектральный синтез, если любое инвариантное подпространство $\mathscr{H} \subseteq \mathscr{F}$ допускает строгий спектральный синтез.

Из теоремы 1.1 и из предложения 1.1 вытекает, что если группа $G$ поэлементно компактная, то в пространстве $C(G)$ справедлив строгий спектральный синтез. Можно доказать, что если LCA-группа $G$ не является поэлементно компактной, то строгий спектральный синтез в пространстве $C(G)$ не справедлив (см. § 3, предложение 3.6). Таким образом, условие поэлементной компактности является необходимым и достаточным условием для справедливости строгого спектрального синтеза в пространстве $C(G)$.

Приведем некоторые примеры поэлементно компактных LCA-групп, которые не являются нульмерными или компактными. Тривиальными примерами являются группы $G$, представимые в виде прямого произведения $G=G_{1} \times G_{2}$, где $G_{1}$ - нульмерная поэлементно компактная, но некомпактная LCA-группа, $G_{2}$ - любая компактная, но не нульмерная абелева группа (примеры нульмерных поэлементно копактных групп см., например, в [4] и в цитированной там литературе).

Известно, что LCA-группа $G$ является нульмерной тогда и только тогда, когда ее двойственная группа $\widehat{G}$ является поэлементно компактной (см. [7; п. 24.18]). Поэтому поэлементно компактные LCA-группы можно охарактеризовать как двойственные группы к нульмерным LCA-группам.

Более конкретные примеры поэлементно компактных LCA-групп могут быть построены по следующей схеме.

Пусть $\left\{G_{i}\right\}_{i \in I}$ - непустое семейство компактных абелевых групп, и для каждого $i \in I$ пусть $H_{i}$ - открытая подгруппа в $G_{i}$, причем $H_{i} \neq G_{i}$. Будем считать, что операции в группах задаются мультипликативно и $e-$ единичный элемент (единица) в любой группе.

Пусть $\widetilde{G}=\prod_{i \in I} G_{i}$ - прямое произведение групп $G_{i}$. Элементами $\widetilde{G}$ являются обобщенные последовательности $x=\left\{x_{i}\right\}_{i \in I}, x_{i} \in G_{i}$.

Пусть $\Lambda$ - произвольное конечное подмножество в $I$, и для каждого $i \in \underset{\widetilde{G}}{\Lambda}$ пусть $U_{i}$ - некоторая окрестность единицы в $G_{i}$. Зададим топологию на $\widetilde{G}$, приняв за открытую базу единицы в $\widetilde{G}$ совокупность множеств вида

$$
U=\prod_{i \in \Lambda} U_{i} \times \prod_{i \notin \Lambda} H_{i} .
$$

Обозначим через $G$ подгруппу группы $\widetilde{G}$, состоящую из всех тех $x=\left\{x_{i}\right\}_{i \in I}$, для которых $x_{i} \notin H_{i}$ возможно лишь для конечного числа индексов из $I$. Рассматриваемая как подпространство группы $\widetilde{G}$ группа $G$ будет топологической абелевой группой, которая называется локальным прямым произведением групп $G_{i}$ относительно открытых подгрупп $H_{i}$. Приведенная конструкция является частным случаем более общего определения локального прямого произведения (см. [7; п. 6.16] или [11]). 
ПРЕДЛОЖЕНИЕ 1.2. Если множество I бесконечно, то построенная выше группа $G$ является некомпактной LCA-группой, все элементы которой компактны.

ДокАЗАТЕЛЬСтво. 1) Пусть $\Lambda$ - конечное подмножество в $I$. Проверим, что множество

$$
G(\Lambda):=\prod_{i \in \Lambda} G_{i} \times \prod_{i \notin \Lambda} H_{i}
$$

является компактной подгруппой в группе $G$.

Заметим, что множество $G(\Lambda)$ будет открытой подгруппой в топологической группе $\widetilde{G}$ и индуцированная из $\widetilde{G}$ топология на $G(\Lambda)$ совпадает с тихоновской топологией произведения топологических пространств. Так как любая открытая подгруппа топологической группы является замкнутой, то открытая подгруппа $H_{i}$ будет замкнутой подгруппой в компактной группе $G_{i}$ и, следовательно, $H_{i}$ является компактной подгруппой в $G_{i}$. Тогда подгруппа $G(\Lambda)$ будет компактным топологическим пространством как тихоновское произведение компактных пространств. Так как $G(\Lambda) \subseteq G$, то $G(\Lambda)$ является компактной подгруппой группы $G$.

Очевидно, что каждый элемент из группы $G$ содержится в некоторой подгруппе $G(\Lambda)$, поэтому группа $G$ поэлементно компактна.

2) Базу окрестностей единицы группы $G$ образуют множества $U$ вида (1.2). Окрестность $U$ содержится в компактной подгруппе $G(\Lambda)$, поэтому ее замыкание компактно. Следовательно, $G$ является локально компактной абелевой группой.

3) Проверим, что группа $G$ некомпактная, если множество $I$ бесконечно.

Так как $H_{i} \neq G_{i}$, то для каждого $i \in I$ можно выбрать элемент $g_{i} \in G_{i}$ такой, что $g_{i} \notin H_{i}$. Для любого $\alpha \in I$ определим обобщенную последовательность $y^{(\alpha)}=\left\{y_{i}^{(\alpha)}\right\}_{i \in I}$, где элемент $y_{i}^{(\alpha)} \in G_{i}$ задается формулой

$$
y_{i}^{(\alpha)}=\left\{\begin{array}{lll}
g_{\alpha} & \text { при } i=\alpha ; \\
e & \text { при } i \neq \alpha .
\end{array}\right.
$$

Тогда $y^{(\alpha)} \in G$ и множество точек $\left\{y^{(\alpha)}, \alpha \in I\right\}$ является дискретным подмножеством группы $G$, так как множества

$$
V_{\alpha}:=\left(G \backslash H_{\alpha}\right) \times \prod_{i \neq \alpha} H_{i}
$$

являются попарно не пересекающимися окрестностями точек $y^{(\alpha)}$. Таким образом, в $G$ имеется бесконечное дискретное подмножество, поэтому топологическая группа $G$ некомпактна.

Напомним, что топологическое пространство $X$ называется нульмерным, если его открыто-замкнутые подмножества образуют базу топологии. Если $X$ хаусдорфово локально компактное пространство, то $X$ нульмерно тогда и только тогда, когда $X$ вполне несвязно, т.е. любое подмножество в $X$, содержащее более одной точки, несвязно (см., например, [7; гл. $1, \S 3]$ ). 
Пусть $G$ - локальное прямое произведение компактных абелевых групп $G_{i}$ относительно открытых подгрупп $H_{i}, i \in I$. Для каждого $\alpha \in I$ пусть

$$
\widetilde{G}_{\alpha}=G_{\alpha} \times \prod_{i \neq \alpha}\{e\} .
$$

Тогда $\widetilde{G}_{\alpha}$ является замкнутой подгруппой в группе $G$ изоморфной (как топологическая группа) группе $G_{\alpha}$.

Предложение 1.2 позволяет строить примеры поэлементно компактных LCAгрупп. При этом, если хотя бы одна из топологических групп $G_{\alpha}, \alpha \in I$, не является нульмерной, то и вся группа $G$ не будет нульмерной. Это вытекает, например, из того, что любое подпространство нульмерного пространства должно быть нульмерным, а подпространство $\widetilde{G}_{\alpha}$ пространства $G$ не является нульмерным. В качестве групп $G_{i}$ можно взять, например, топологические прямые произведения $G_{i}=K_{i} \times \mathbb{Z}_{2}$, где $K_{i}$ - произвольная компактная группа, $\mathbb{Z}_{2}=\{1,-1\}$ - мультипликативная циклическая группа из двух элементов, снабженная дискретной топологией. Пусть $H_{i}=K_{i} \times\{1\}$, тогда $H_{i}$ является открытой подгруппой в компактной группе $G_{i}$. Если группа $K_{i}$ не нульмерная, то и группа $G_{i}$ не будет нульмерной.

Кратко изложим схему доказательства теоремы 1.1. Основным инструментом доказательства теоремы 1.1 являются функции Брюа-Шварца на LCAгруппах. Для любой LCA-группы $G$ пусть $\mathscr{S}(G)$ - множество функций БрюаШварца на группе $G$. В $\S 2$ приводятся необходимые сведения о функциях Брюа-Шварца и описывается строение этих функций на поэлементно компактных и нульмерных LCA-группах. Для любой LCA-группы $G$ множество $\mathscr{S}(G)$ является топологической алгеброй относительно поточечного умножения функций. В $\S 2$ описываются замкнутые идеалы в алгебре $\mathscr{S}(G)$ для случая, когда $G$ - нульмерная LCA-группа. Доказано, что любой замкнутый идеал в алгебре $\mathscr{S}(G)$ определяется множеством его нулей (т.е. множеством общих нулей функций из идеала). В $\S 3$ с помощью перехода к двойственному пространству $C^{\prime}(G)$ и с помощью преобразования Фурье задача о спектральном синтезе в $C(G)$ сводится к задаче об описании замкнутых идеалов в алгебpe $\mathscr{S}(\widehat{G})$ множествами их нулей. Если группа $G$ поэлементно компактная, то двойственая группа $\widehat{G}$ нульмерная и теорема 1.1 выводится из результатов $\S 2$.

\section{§ 2. Функции Брюа-Шварца}

Всюду в дальнейшем $G$ - LCA-группа. Элементы группы $G$ будут в дальнейшем обозначаться $x, y$ и так далее, пусть $d x$ (соответственно $d y$ и так далее) элемент меры Хаара на группе $G$. Через $\widehat{G}$ будем обозначать двойственную группу к группе $G$. Элементами $\widehat{G}$ являются характеры группы $G$, которые обычно будем обозначать через $\chi$. Пусть $d \chi$ - элемент меры Хаара на группе $\widehat{G}$. Если не оговорено противное, то операции в группах задаются мультипликативно, $e$ - единичный элемент группы. 
Все топологические пространства (и, в частности, топологические группы) предполагаются хаусдорфовыми. Под окрестностью в топологическом пространстве всегда понимается открытая окрестность. Если $A$ - подмножество топологического пространства, то через $[A]$ обозначается замыкание множества $A$. Если $X-$ множество с мерой $\mu$, то $L^{p}(X)=L^{p}(X, d \mu), 1 \leqslant p \leqslant \infty,-$ лебегово пространство с нормой

$$
\|f\|_{L^{p}(X)}=\|f\|_{p}:= \begin{cases}\left(\int_{X}|f(x)|^{p} d \mu(x)\right)^{1 / p} & \text { при } 1 \leqslant p<\infty ; \\ \operatorname{supp\operatorname {vrai}}|f(x)|_{x \in X} & \text { при } p=\infty .\end{cases}
$$

Функции из лебеговых пространств рассматриваются с точностью до значений на множестве меры нуль. Если $X$ - LCA-группа или ее подмножество, то в качестве меры в лебеговых пространствах всегда берется мера Хаара.

Пространство $L^{1}(G)$ является коммутативной банаховой алгеброй относительно свертки

$$
\left(f_{1} * f_{2}\right)(x):=\int f_{1}\left(x y^{-1}\right) f_{2}(y) d y, \quad f_{1}, f_{2} \in L^{1}(G) .
$$

Для любой функции $f \in L^{1}(G)$ ее преобразованием Фуръе называется функция $\widehat{f}$ на двойственной группе $\widehat{G}$, которая определяется формулой

$$
\widehat{f}(\chi):=\int_{G} f(x) \overline{\chi(x)} d x, \quad \chi \in \widehat{G} .
$$

Отображение $\Phi: f \mapsto \widehat{f}$ также будем называть преобразованием Фурье.

Легко проверяются следующие свойства преобразования Фурье:

$$
\begin{gathered}
|\widehat{f}(\chi)| \leqslant\|f\|_{1}, \quad f \in L^{1}(G), \quad \chi \in \widehat{G} ; \\
\Phi\left(f_{1} * f_{2}\right)=\Phi\left(f_{1}\right) \Phi\left(f_{2}\right), \quad f_{1}, f_{2} \in L^{1}(G) ; \\
\widehat{\tau_{y} f}(\chi)=\chi(y) \widehat{f}(\chi), \quad f \in L^{1}(G), \quad y \in G, \quad \chi \in \widehat{G},
\end{gathered}
$$

где $\left(\tau_{y} f\right)(x)=f(x y)$.

Если $S$ - подмножество в $G$, то через $\langle S\rangle$ обозначается подгруппа, порожденная множеством $S$. В частности, если $U$ - симметричная окрестность единицы (т.е. $U^{-1}=U$ ), то $\langle U\rangle=\bigcup_{k=1}^{\infty} U^{k}$. В этом случае множество $\langle U\rangle$ будет открыто-замкнутой подгруппой в группе $G$ (см. [7; теорема 5.7]). Группа $G$ называется компактно порожденной, если в $G$ существует окрестность единицы $U$ с компактным замыканием такая, что $G=\langle U\rangle$. Фундаментальным результатом, описывающим строения компактно порожденных LCA-групп, является следующая теорема (см. [7; теорема 9.8]).

Теорема 2.1. Любая компактно порожденная LCA-группа $G$ топологически изоморфна группе $\mathbb{R}^{a} \times \mathbb{Z}^{b} \times K$, где а и $b$ - неотрицательные челье числа, $K$ - компактная абелева группа. 
ПРеДЛОЖеНИЕ 2.1. Любая компактно порожденная поэлементно компактная LCA-группа $G$ является компактной топологической группой.

ДокАЗАтельство. По теореме 2.1 группа $G$ изоморфна группе $\mathbb{R}^{a} \times \mathbb{Z}^{b} \times K$, где $a, b \in \mathbb{Z}_{+}, K$ - компактная группа. Так как группа $G$ поэлементно компактная, то $a=b=0$, т.е. $G$ изоморфна компактной группе $K$.

ПРЕДЛОЖЕНИЕ 2.2. 1) Если $G$ - поэлементно компактная LCA-группа, то для любого компактного подмножества $P \subseteq G$ существует компактная открытая подгруппа группы $G$, содержащая множество $P$.

2) Если $G$ - нульмерная LCA-группа, то для любого компактного подмножества $P \subseteq G$ существует симметричная компактная открытая окрестность единиць, содержащая множество $P$.

ДокАЗАтЕЛЬство. 1) Без ограничения общности можно считать, что $e \in P$. Пусть $V$ - произвольная окрестность точки $e$ с компактным замыканием, тогда множество $U=(V P) \cup(V P)^{-1}$ будет симметричной окрестностью точки $e$ с компактным замыканием и $P \subseteq U$. Подмножество $H:=\langle U\rangle$ будет открыто-замкнутой подгруппой в $G$, содержащей $P$. Так как $H$ является компактно порожденной поэлементно компактной LCA-группой, то по предложению $2.1 H$ является компактной подгруппой.

2) Так как $G$ - нульмерная LCA-группа, то базу топологии в $G$ образуют компактные открытые подмножества. Пусть $V$ - произвольная компактная открытая окрестность единицы в $G$. Без ограничения общности можно считать, что $e \in P$. Тогда множество $U=(V P) \cup(V P)^{-1}$ будет компактной открытой симметричной окрестностью единицы и $P \subseteq U$.

В [12] Ф. Брюа определил аналоги быстроубывающих функций Шварца для любой LCA-группы. Эти функции называются функциями Брюа-Шварца. Другое описание функций Брюа-Шварца было получено М. Осборном в [13]. Мы рассмотрим некоторые свойства функций Брюа-Шварца, которые будут использоваться в дальнейшем. Используемые при этом определения и обозначения близки к определениям и обозначениям из [13].

Через $\mathfrak{V}(G)$ будем обозначать множество всех симметричных окрестностей единицы с компактным замыканием в группе $G$. Для любого $V \in \mathfrak{V}(G)$ обозначим через $\mathscr{A}_{V}(G)$ множество всех функций $f \in L^{\infty}(G)$, удовлетворяющих условию: для любого $n \in \mathbb{Z}_{+}=\{0,1,2, \ldots\}$ существует число $M_{n}>0$ такое, что для всех $k \in \mathbb{N}$ справедливо неравенство

$$
\left\|\left.f\right|_{G \backslash V^{k}}\right\|_{\infty} \leqslant M_{n} k^{-n} .
$$

Множество $\mathscr{A}_{V}(G)$ является локально выпуклым пространством с топологией, порожденной семейством полунорм (даже норм):

$$
\nu_{V, n}(f):=\|f\|_{\infty}+\sup _{k \in \mathbb{N}} k^{n}\left\|\left.f\right|_{G \backslash V^{k}}\right\|_{\infty}, \quad f \in \mathscr{A}_{V}(G), \quad n \in \mathbb{Z}_{+} .
$$

Очевидно, что если $V_{1} \subseteq V_{2}\left(V_{1}, V_{2} \in \mathfrak{V}(G)\right)$, то $\mathscr{A}_{V_{1}}(G) \subseteq \mathscr{A}_{V_{2}}(G)$, причем вложение непрерывное, так как $\nu_{V_{1}, n}(f) \leqslant \nu_{V_{2}, n}(f)$ при $f \in \mathscr{A}_{V_{1}}(G)$. 
Пространство

$$
\mathscr{A}(G):=\bigcup_{V \in \mathfrak{V}(G)} \mathscr{A}_{V}(G)
$$

снабдим топологией индуктивного предела локально выпуклых пространств $\mathscr{A}_{V}(G)$, т.е. топология в $\mathscr{A}(G)$ совпадает со слабейшей локально выпуклой топологией, для которой все вложения $\mathscr{A}_{V}(G) \subseteq \mathscr{A}(G)$ непрерывны.

Приведем некоторые свойства пространства $\mathscr{A}(G)$ (см. [13]).

$(\mathscr{A} 1)$ Если $f \in \mathscr{A}_{V}(G)$, то $f(x)=0$ п.в. на множестве $G \backslash\langle V\rangle$.

$(\mathscr{A} 2) \mathscr{A}(G) L^{\infty}(G) \subseteq \mathscr{A}(G)$, и, в частности, $\mathscr{A}(G)$ является алгеброй относительно поточечного умножения функций.

$(\mathscr{A} 3) \mathscr{A}(G) \subseteq L^{1}(G)$, и если $f \in \mathscr{A}_{V}(G)$, то для любого $n \in \mathbb{Z}_{+}$существует число $M_{n}^{\prime}>0$ такое, что при всех $k \in \mathbb{N}$ справедливо неравенство

$$
\left\|\left.f\right|_{G \backslash V^{k}}\right\|_{1} \leqslant M_{n}^{\prime} k^{-n} .
$$

$(\mathscr{A} 4) \mathscr{A}(G)$ является коммутативной алгеброй относительно свертки функций.

ОПРЕДЕЛЕНИЕ 2.1 (см. [13]). Функция $f$ называется функиией Брюа-Швариа, если $f \in \mathscr{A}(G)$ и $\widehat{f} \in \mathscr{A}(\widehat{G})$, где $\widehat{f}$ - преобразование Фурье функции $f$.

Через $\mathscr{S}(G)$ обозначим пространство Брюа-Шварца на группе $G$, т.е. множество всех функций Брюа-Шварца на группе $G$.

Зададим топологию на пространстве $\mathscr{S}(G)$. Для любых $V \in \mathfrak{V}(G)$ и $W \in$ $\mathfrak{V}(\widehat{G})$ обозначим через $\mathscr{S}(V, W)$ множество всех функций $f$, удовлетворяющих условиям $f \in \mathscr{A}_{V}(G)$ и $\widehat{f} \in \mathscr{A}(\widehat{G})$. Множество $\mathscr{S}(V, W)$ является локально выпуклым пространством с топологией, порожденной семейством полунорм

$$
N_{V, W, m, n}(f):=\nu_{V, m}(f)+\nu_{W, n}(\widehat{f}), \quad m, n \in \mathbb{Z}_{+} .
$$

Пространство $\mathscr{S}(G)$ является объединением пространств $\mathscr{S}(V, W)$ по всем $V \in$ $\mathfrak{V}(G)$ и $W \in \mathfrak{V}(\widehat{G})$ и снабжается топологией индуктивного предела этих локально выпуклых пространств.

Отметим некоторые свойства функций Брюа-Шварца (см. [13]).

$(\mathscr{S} 1) \mathscr{S}(G) \subseteq C(G)$.

$(\mathscr{S} 2)$ Преобразование Фурье устанавливает изоморфизм топологических векторных пространств $\mathscr{S}(G)$ и $\mathscr{S}(\widehat{G})$.

$(\mathscr{S} 3) \mathscr{S}(G)$ является топологической алгеброй относительно поточечного умножения функций и топологической алгеброй относительно свертки функций.

Для любой функции $f$ через $\operatorname{supp}(f)$ будем обозначать носитель функции $f$. Пусть $C_{\mathrm{c}}(G)$ - множество непрерывных функций на $G$ с компактным носителем. По определению

$$
\mathscr{D}(G):=\mathscr{S}(G) \cap C_{\mathrm{c}}(G) .
$$

Функции из пространства $\mathscr{D}(G)$ называются гладкими функциями с компактным носителем на группе $G$. 
Пусть $L_{\mathrm{c}}^{\infty}(G)$ - множество всех функций из класса $L^{\infty}(G)$ с компактным носителем. Определим топологию на пространстве $L_{\mathrm{c}}^{\infty}(G)$. Для любого $V \in \mathfrak{V}(G)$ обозначим через $L_{\mathrm{c}}^{\infty}(V)$ множество функций $f \in L_{\mathrm{c}}^{\infty}(G)$, удовлетворяющих условию $\operatorname{supp}(f) \subseteq[V]$, где $[V]$ - замыкание множества $V$. Снабженное нормой $\|\cdot\|_{\infty}$ множество $L_{\mathrm{c}}^{\infty}(G)$ является банаховым пространством. Пространство

$$
L_{\mathrm{c}}^{\infty}(G)=\bigcup_{V \in \mathfrak{V}(G)} L_{\mathrm{c}}(V)
$$

снабжается топологией индуктивного предела нормированных пространств $L_{\mathrm{c}}^{\infty}(V)$ и становится локально выпуклым пространством. Пространство $C_{\mathrm{c}}(G)$ является замкнутым линейным подпространством ТВП $L_{\mathrm{c}}^{\infty}(G)$ и $C_{\mathrm{c}}(G)$ является локально выпуклым пространством с топологией, индуцированной топологией пространства $L_{\mathrm{c}}^{\infty}(G)$.

ПреДЛОЖеНИЕ 2.3. 1) Для любой LCA-группъ $G$

$$
L_{\mathrm{c}}^{\infty}(G) \subseteq \mathscr{A}(G)
$$

причем вложение непрерывно.

2) Если $G$ - поэлементно компактная LCA-группа, то

$$
\mathscr{A}(G)=L_{\mathrm{c}}^{\infty}(G)
$$

как топологические векторные пространства.

ДокАЗАтельство. 1) Пусть $f \in L_{\mathrm{c}}^{\infty}(W), W \in \mathfrak{V}(G), U-$ произвольная симметричная окрестность единицы с компактным замыканием. Возьмем $V:=$ $U[W]$, тогда $V \in \mathfrak{V}(G)$ и $\operatorname{supp}(f) \subseteq V$. Так как $V \subseteq V^{k}$ для любого $k \in \mathbb{N}$, то $\left\|\left.f\right|_{G \backslash V^{k}}\right\|_{\infty}=0$, следовательно, $f \in \mathscr{A}_{V}(G)$ и из определения полунормы $\nu_{V, n}$ (см. (2.6)) вытекает, что

$$
\nu_{V, n}(f)=\|f\|_{\infty} \quad \forall n \in \mathbb{N} .
$$

Таким образом, доказано, что для любой окрестности $W \in \mathfrak{V}(G)$ существует окрестность $V \in \mathfrak{V}(G)$ такая, что

$$
L_{\mathrm{c}}^{\infty}(W) \subseteq \mathscr{A}_{V}(G)
$$

причем вложение непрерывно. Из (2.13) вытекает (2.10), а из определения топологии индуктивного предела следует, что вложение (2.10) непрерывно.

2) Обозначим через $\Lambda$ множество всех компактных открытых подгрупп группы $G$. Если $V \in \Lambda$ и $f \in \mathscr{A}_{V}(G)$, то по свойству $(\mathscr{A} 1) \operatorname{supp}(f) \subseteq\langle V\rangle=V$, следовательно, $f \in L_{\mathrm{c}}^{\infty}(G)$. Рассуждая как в части 1$)$ доказательства, получим, что справедливо равенство (2.12). Таким образом, при $V \in \Lambda$ имеется вложение

$$
\mathscr{A}_{V}(G) \subseteq L_{\mathrm{c}}^{\infty}(V)
$$

причем это вложение непрерывно. 
Если группа $G$ поэлементно компактна, то по предложению 2.2 для любой окрестности $U \in \mathfrak{V}(G)$ найдется содержащая ее компактная открытая подгруппа $V$, поэтому

$$
\mathscr{A}(G)=\bigcup_{V \in \Lambda} \mathscr{A}_{V}(G) \subseteq \bigcup_{V \in \Lambda} L_{\mathrm{c}}^{\infty}(V)=L_{\mathrm{c}}^{\infty}(G),
$$

т.е.

$$
\mathscr{A}(G) \subseteq L_{\mathrm{c}}^{\infty}(G),
$$

причем это вложение непрерывно. Из (2.10) и (2.15) вытекает равенство (2.11).

С учетом свойства $(\mathscr{S} 1)$, из предложения 2.3 вытекает следующее.

СледСтвие 2.1. Если $G$ - поэлементно компактная LCA-группа, то $\mathscr{S}(G)=\mathscr{D}(G)$.

Пусть $G$ - произвольная LCA-группа. Если $H$ - замкнутая подгруппа группы $G$, то аннулятором $H$ в двойственной группе $\widehat{G}$ называется множество

$$
A(\widehat{G}, H):=\{\chi \in \widehat{G}: \chi(x)=1 \forall x \in H\} .
$$

Множество $A(\widehat{G}, H)$ является замкнутой подгруппой группы $\widehat{G}$. Аналогично для любой замкнутой подгруппы Г группы $G$ определяется ее аннулятор в группе $G$ :

$$
A(G, \Gamma):=\{x \in G: \chi(x)=1 \forall \chi \in \Gamma\} .
$$

Приведем некоторые свойства двойственных групп и аннуляторов подгрупп.

ПРЕДЛОЖЕНИЕ 2.4. 1) Если $H$ - замкнутая подгруппа группљ $G$, то

$$
A(G, A(\widehat{G}, H))=H .
$$

2) Подгруппа $H$ является компактной открытой подгруппой группљ $G$ тогда и только тогда, когда подгруппа $A(\widehat{G}, H)$ является компактной открытой nодгрупппой группы $\widehat{G}$.

3) Группа $G$ поэлементно компактная тогда и только тогда, когда ее двойственная группа $\widehat{G}$ нульмерная.

Доказательство п. 1) см. в [7; п. 24.10], п. 2) - в. [7; п. 23.29], п. 3) - в [7; п. 24.18].

Пусть $X$ - топологическое пространство. Функция $f$ на $X$ называется локально постоянной, если для любой точки $x \in X$ найдется окрестность $U=$ $U(x)$ этой точки, в которой функция $f$ постоянная. Если $G$ - топологическая группа, то функцию $f$ на $G$ будем называть равномерно локально постоянной, если в $G$ существует такая окрестность единицы $U$, что $f(x y)=f(x)$ для всех $x \in G, y \in U$. Будем обозначать множество всех равномерно локально постоянных функций на $G$ через $\mathscr{C}(G)$.

Как и ранее, всюду $G$ - LCA-группа. 
ПредлОжениЕ 2.5. Если группа $G$ нульмерная, то справедливо равенство

$$
\mathscr{S}(G)=\mathscr{C}(G) \cap \mathscr{A}(G),
$$

т.е. функиия $f$ является функиией Брюа-Шварца тогда и только тогда, когда функиия $f$ равномерно локально постоянная и принадлежит классу $\mathscr{A}(G)$.

ДоказАтельство. 1) Пусть $f \in \mathscr{S}(G)$, тогда по определению $f \in \mathscr{A}(G)$ и $\widehat{f} \in \mathscr{A}(\widehat{G})$. Так как группа $G$ нульмерная, то двойственная группа $\widehat{G}$ будет поэлементно компактной (см. предложение 2.4,3)). По предложению 2.3 $\mathscr{A}(\widehat{G})=L_{\mathrm{c}}^{\infty}(\widehat{G})$, поэтому найдется компактное подмножество $P \subseteq \widehat{G}$, для которого $\operatorname{supp}(\widehat{f}) \subseteq P$. По предложению 2.2 существует компактная открытая подгруппа $H$ группы $\widehat{G}$, содержащая множество $P$.

Пусть $U=A(G, H)$ - аннулятор подгруппы $H$ в группе $G$. Тогда $U$ является компактной открытой подгруппой группы $G$ (см. предложение $2.4,2)$ ). Проверим, что

$$
f(x y)=f(x) \quad \forall x \in G, y \in U .
$$

Пусть $\left(\tau_{y} f\right)(x)=f(x y)$, тогда по свойству $(2.4)$

$$
\widehat{\tau_{y} f}(\chi)=\chi(y) \widehat{f}(\chi) \quad \forall \chi \in \widehat{G} .
$$

Так как $\operatorname{supp}(\widehat{f}) \subseteq H$, то при $\chi \notin H$ будет $\widehat{f}(\chi)=0$, следовательно, $\chi(y) \widehat{f}(\chi)=$ $\widehat{f}(\chi)$. Если $\chi \in H$, то из того, что $y \in U=A(G, H)$, вытекает, что $\chi(y)=1$, поэтому $\chi(y) \widehat{f}(\chi)=\widehat{f}(\chi)$. В результате $\widehat{\tau_{y} f}(\chi)=\widehat{f}(\chi)$ для любого $\chi \in \widehat{G}$, откуда следует (2.16). Следовательно, $f \in \mathscr{C}(G)$ и тем самым доказано включение

$$
\mathscr{S}(G) \subseteq \mathscr{C}(G) \cap \mathscr{A}(G) .
$$

2) Обратно, пусть $f \in \mathscr{C}(G) \cap \mathscr{A}(G)$. Так как $f \in \mathscr{C}(G)$, то существует окрестность единицы $U \subseteq G$ такая, что выполняется условие (2.16). Так как на нульмерной LCA-группе базу окрестностей единицы образуют компактные открытые подгруппы (см. [8; гл. 3, § 22], то без ограничения общности можно считать, что $U$ - компактная открытая подгруппа группы $G$. Пусть $H=A(\widehat{G}, U)$. Тогда $H$ будет компактной открытой подгруппой группы $\widehat{G}$.

Проверим, что $\operatorname{supp}(\widehat{f}) \subseteq H$. Если $\chi \notin H$, то найдется элемент $y \in U$, для которого $\chi(y) \neq 1$. Из равенства $\tau_{y} f=f$ следует, что $\widehat{\tau_{y} f}(\chi)=\widehat{f}(\chi)$, т.е. $\chi(y) \widehat{f}(\chi)=\widehat{f}(\chi)$, откуда вытекает, что $\hat{f}(\chi)=0$.

Так как $\widehat{f} \in L^{\infty}(\widehat{G})$ и $\operatorname{supp}(\widehat{f}) \subseteq H$, то $\widehat{f} \in L_{\mathrm{c}}^{\infty}(\widehat{G})=\mathscr{A}(\widehat{G})$. Окончательно, $f \in \mathscr{A}(G)$ и $\widehat{f} \in \mathscr{A}(\widehat{G})$, т.е. $f \in \mathscr{S}(G)$, и тем самым доказано включение

$$
\mathscr{C}(G) \cap \mathscr{A}(G) \subseteq \mathscr{S}(G) .
$$

ПредлОЖениЕ 2.6. Если группа $G$ нульмерная, то любая локалъно постоянная функиия на $G$ с компактным носителем принадлежит классу $\mathscr{S}(G)$.

ДокАзАТЕльство. Пусть $f$ - локально постоянная функция с компактным носителем на нульмерной группе $G$. Так как $f \in L_{\mathrm{c}}^{\infty}(G)$, то $f \in \mathscr{A}(G)$ по предложению 2.3. По предложению 2.5 , чтобы доказать, что $f \in \mathscr{S}(G)$, достаточно показать, что функция $f$ является равномерно локально постоянной. 
Пусть $P=\operatorname{supp}(f)$. Так как функция $f$ локально постоянная, то $P$ является компактным открытым подмножеством в $G$. Пусть $\Lambda$ - множество компактных открытых подгрупп группы $G$. Так как $G$ - нульмерная LCA-группа, то множество $\Lambda$ образует базу окрестностей единицы группы $G$. Для любой точки $a \in G$ базу окрестностей точки $a$ образуют множества вида $a V$, где $V \in \Lambda$.

Так как множество $P$ открыто, а функция $f$ локально постоянная, то для каждой точки $a \in P$ существует окрестность единицы $V_{a} \in \Lambda$ такая, что $a V_{a} \subseteq P$ и функция $f$ постоянна на множестве $a V_{a}$. Семейство $\left\{a V_{a}, a \in P\right\}$ является открытым покрытием множества $P$, а так как множество $P$ компактное, то из него можно выделить конечное подпокрытие, т.е. найдутся точки $a_{1}, \ldots, a_{m} \in P$ такие, что

$$
P=\bigcup_{j=1}^{m} a_{j} V_{a_{j}} .
$$

Пусть

$$
U=\bigcap_{j=1}^{m} V_{a_{j}},
$$

тогда $U \in \Lambda$. Проверим, что для функции $f$ выполняется соотношение $(2.16)$. Рассмотрим два случая.

1) Пусть $x \notin P$, тогда $f(x)=0$. Если $y \in U$ и $x y \in P$, то $x y \in a_{j} V_{a_{j}}$ для некоторого $j$. Так как $V_{a_{j}}$ - подгруппа группы $G$ и $y \in U \subseteq V_{a_{j}}$, то $x \in a_{j} y^{-1} V_{a_{j}}=a_{j} V_{a_{j}}$, поэтому $x \in P$, что невозможно. Следовательно, $x y \notin P$ и $f(x y)=0$.

2) Пусть $x \in P$, тогда $x \in a_{j} V_{a_{j}}$ для некоторого $j$. Так как $y \in U \subseteq V_{a_{j}}$, то $x y \in a_{j} y V_{a_{j}}=a_{j} V_{a_{j}}$. Обе точки $x$ и $x y$ принадлежат множеству $a_{j} V_{a_{j}}$, на котором функция $f$ постоянная, следовательно, $f(x y)=f(x)$.

СлЕДСТвиЕ 2.2. Если $U$ - компактное открытое подмножество нулъмерной группљ $G$, то характеристическая функиия $I_{U}$ принадлежит множеству $\mathscr{S}(G)$, где

$$
I_{U}(x)= \begin{cases}1, & x \in U ; \\ 0, & x \notin U .\end{cases}
$$

Для любой LCA-группы $G$ пространство $\mathscr{S}(G)$ является топологической алгеброй относительно поточечного умножения функций и топологической алгеброй относительно свертки функций. Чтобы различать эти топологические алгебры, будем использовать обозначения $\mathscr{S}_{\text {mult }}(G)$, когда $\mathscr{S}(G)$ рассматривается как топологическая алгебра относительно поточечного умножения, и $\mathscr{S}_{\text {conv }}(G)$, когда $\mathscr{S}(G)$ рассматривается как топологическая алгебра относительно свертки.

Пусть $X$ - произвольное топологическое пространство, $\mathscr{F}$ - некоторое множество, состоящее из непрерывных функций на $X, \mathscr{W}$ - подмножество в $\mathscr{F}$. Обозначим через $N(\mathscr{W} ; X)$ множество общих нулей всех функций из $\mathscr{W}$, т.е.

$$
N(\mathscr{W} ; X):=\{x \in X: f(x)=0 \forall f \in \mathscr{W}\} .
$$


Так как все функции из $\mathscr{F}$ непрерывны, то множество $N(\mathscr{W} ; X)$ является замкнутым подмножеством пространства $X$. Будем называть $N(\mathscr{W} ; X)$ нулевым множеством подмножества $\mathscr{W}$.

Для любого замкнутого подмножества $A \subseteq X$ обозначим через $\mathscr{V}(A ; \mathscr{F})$ множество всех функций $f \in \mathscr{F}$ таких, что $f(x)=0$ для любой точки $x \in A$.

В частности, возможен случай, когда $X=G$ - произвольная LCA-группа и $\mathscr{F}=\mathscr{S}(G)$ - множество функций Брюа-Шварца. Если $A$ - замкнутое подмножество в $G$, то пусть $\mathscr{V}(A):=\mathscr{V}(A ; \mathscr{S}(G))$. Очевидно, что $\mathscr{V}(A)$ является замкнутым идеалом в топологической алгебре $\mathscr{S}_{\text {mult }}(G)$.

Лемма 2.1. Пусть $G$ - нульмерная LCA-группа. Если $A$ и $B$-различные замкнутые подмножества в $G$, то $\mathscr{V}(A) \neq \mathscr{V}(B)$.

Доказательство. Если $A \neq B$, то $A \backslash B \neq \varnothing$ или $B \backslash A \neq \varnothing$. Для определенности пусть $A \backslash B \neq \varnothing$, тогда существует точка $x_{0} \in A$ и $x_{0} \notin B$. Так как пространство $G$ локально компактное и нульмерное, то найдется открытое компактное подмножество $U$ такое, что $x_{0} \in U$ и $U \cap B=\varnothing$. По следствию 2.2 характеристическая функция $I_{U}$ принадлежит классу $\mathscr{S}(G)$. Тогда $I_{U} \in \mathscr{V}(B)$ и $I_{U} \notin \mathscr{V}(A)$, следовательно, $\mathscr{V}(A) \neq \mathscr{V}(B)$.

Из следующей теоремы вытекает, что идеалами вида $\mathscr{V}(A), A$ - замкнутое подмножество в $G$, исчерпываются все замкнутые идеалы в топологической алгебре $\mathscr{S}_{\text {mult }}(G)$, если $G$ - нульмерная группа.

Теорема 2.2. Если $G$ - нулвмерная LCA-группа, то для любого замкнутого идеала $\mathscr{I}$ топологической алгебры $\mathscr{S}_{\text {mult }}(G)$ справедливо равенство

$$
\mathscr{I}=\mathscr{V}(N ; \mathscr{S}(G))
$$

где $N=N(\mathscr{I} ; G)$ - нулевое множество идеала $\mathscr{I}$.

Предварительно приведем вспомогательный результат, необходимый для доказательства теоремы 2.2. Пусть $X$ - произвольное топологическое пространство, $\mathscr{L}(X)$ - множество всех локально постоянных функций на $X$. Векторное пространство $\mathscr{L}(X)$ является алгеброй относительно поточечного умножения функций.

ТеОрема 2.3. Пусть $X$ - нулвмерное компактное топологическое пространство; тогда для любого идеала $\mathscr{W}$ алгебры $\mathscr{L}(X)$ справедливо равенство

$$
\mathscr{W}=\mathscr{V}(N ; \mathscr{L}(X))
$$

где $N=N(\mathscr{W} ; X)$ - нулевое множество идеала $\mathscr{W}$.

ДокАЗАТЕльство. Теорема является частным случаем теоремы 2.1 в [4], где рассматривался более общий случай, когда $X$ - произвольное нульмерное локально компактное пространство, а вместо алгебры $\mathscr{L}(X)$ рассматривалась алгебра локально постоянных функций на $X$ с компактным носителем. 
ДокАЗАТЕЛЬСтво ТЕОРЕмы 2.2.1) Пусть $G$ - нульмерная LCA-группа, $\mathscr{I}$ замкнутый идеал в алгебре $\mathscr{S}_{\text {mult }}(G), N=N(\mathscr{I} ; G)$ - нулевое множество идеала $\mathscr{I}$. Очевидно, что $\mathscr{I} \subseteq \mathscr{V}(N ; \mathscr{S}(G))=\mathscr{V}(N)$. Нужно доказать обратное включение.

Пусть $f \in \mathscr{V}(N)$. Так как $f \in \mathscr{S}(G)$, то существует подмножество $V \in \mathfrak{V}(G)$ такое, что $f \in \mathscr{A}_{V}(G)$. С учетом п. 2) предложения 2.2 можно считать, что $V-$ компактная открытая окрестность единицы в $G$. Из предложения 2.5 следует, что функция $f$ равномерно локально постоянная, т.е. существует компактная открытая подгруппа $U$ группы $G$ такая, что

$$
f(x y)=f(x) \quad \forall x \in G, y \in U .
$$

Заменив $V$ на $V U$, можно считать, что $U \subseteq V$ и $V U=V$.

Пусть $X_{r}:=V^{r}, r \in \mathbb{N}$. Множества $X_{r}$ образуют возрастающее семейство компактных открытых подмножеств пространства $G$, причем

$$
\begin{gathered}
X_{r} U=X_{r} \quad \forall r \in \mathbb{N}, \\
\operatorname{supp}(f) \subseteq\langle V\rangle=\bigcup_{r=1}^{\infty} X_{r} .
\end{gathered}
$$

Пусть $I_{r}:=I_{X_{r}}-$ характеристическая функция множества $X_{r}$ и $f_{r}:=f I_{r}$. По следствию $2.2 I_{r} \in \mathscr{S}(G)$ и, следовательно, $f_{r} \in \mathscr{S}(G)$. Далее, в п. 2) доказательства, будет показано, что $f_{r} \in \mathscr{I}$ для любого $r \in \mathbb{N}$, а в п. 3) будет показано, что $f_{r} \rightarrow f$ при $r \rightarrow \infty$ в пространстве $\mathscr{S}(G)$. Так как $\mathscr{I}$ является замкнутым подмножеством в $\mathscr{S}(G)$, то из этого будет следовать, что $f \in \mathscr{I}$ и, тем самым, будет доказано включение $\mathscr{V}(N) \subseteq \mathscr{I}$.

2) Каждое множество $X_{r}, r \in \mathbb{N}$, является нульмерным компактным пространством с индуцированной из $G$ топологией. Если $g$ - локально постоянная функция на $X_{r}$, то будем считать, что $g$ продолжена нулем на $G \backslash X_{r}$. Продолженную функцию будем также обозначать через $g$; тогда по предложению 2.6 $g \in \mathscr{I}(G)$. Таким образом, можно считать, что $\mathscr{L}\left(X_{r}\right) \subseteq \mathscr{S}(G)$.

Пусть $\mathscr{I}_{r}:=\mathscr{I} \cap \mathscr{L}\left(X_{r}\right)$ (т.е. $\mathscr{I}_{r}$ состоит из всех функций из $\mathscr{I}$ с носителем, содержащимся в $\left.X_{r}\right), N_{r}:=N \cap X_{r}$. Очевидно, что множество $\mathscr{I}_{r}$ является идеалом в алгебре $\mathscr{L}\left(X_{r}\right)$. Проверим, что $N\left(\mathscr{I}_{r} ; X_{r}\right)=N_{r}$. Включение $N_{r} \subseteq$ $N\left(\mathscr{I}_{r} ; X_{r}\right)$ очевидно. Если $x \in X_{r}$ и $x \notin N_{r}$, то существует функция $g \in \mathscr{I}$ такая, что $g(x) \neq 0$. Тогда функция $\tilde{g}=g I_{r}$ принадлежит идеалу $\mathscr{I}_{r}$ и $\tilde{g}(x)=$ $g(x) \neq 0$, следовательно, $x \notin N\left(\mathscr{I}_{r} ; X_{r}\right)$. Таким образом, $N_{r}=N\left(\mathscr{I}_{r} ; X_{r}\right)$.

По теореме 2.3 справедливо равенство $\mathscr{I}_{r}=\mathscr{V}\left(N_{r} ; \mathscr{L}\left(X_{r}\right)\right)$. Как и раньше, пусть $f \in \mathscr{V}(N), f_{r}=f I_{r}$; тогда $f_{r} \in \mathscr{V}\left(N_{r} ; \mathscr{L}\left(X_{r}\right)\right)$, откуда вытекает, что $f_{r} \in \mathscr{I}_{r}$, а так как $\mathscr{I}_{r} \subseteq \mathscr{I}$, то $f_{r} \in \mathscr{I}$.

3) Чтобы доказать, что $f_{r} \rightarrow f$ при $r \rightarrow \infty$ в пространстве $\mathscr{S}(G)$, достаточно доказать, что $f_{r} \rightarrow f$ в пространстве $\mathscr{A}(G)$ и $\widehat{f}_{r} \rightarrow \widehat{f}$ в пространстве $\mathscr{A}(\widehat{G})$. Так как $f \in \mathscr{A}$, то для $f$ выполняется условие (2.5). Выпишем это условие, заменив $n$ на $(n+1)$ : для любого $n \in \mathbb{Z}_{+}$существует число $M_{n+1}>0$ такое, что для всякого $k \in \mathbb{N}$ справедливо неравенство

$$
\left\|\left.f\right|_{G \backslash V^{k}}\right\|_{\infty} \leqslant M_{n+1} k^{-n-1} .
$$


Пусть $g_{r}=f-f_{r}$, тогда

$$
g_{r}(x)=\left\{\begin{array}{lll}
0 & \text { при } & x \in V^{r} \\
f(x) & \text { при } & x \in G \backslash V^{r} .
\end{array}\right.
$$

Из (2.21) и (2.22) следует, что

$$
\left\|g_{r}\right\|_{\infty} \leqslant M_{n+1} r^{-n-1} .
$$

Так как $\left|g_{r}(x)\right| \leqslant|f(x)|$, то из (2.21) вытекает, что для любых $k, r \in \mathbb{N}$ справедливо неравенство

$$
\left\|\left.g_{r}\right|_{G \backslash V^{k}}\right\|_{\infty} \leqslant M_{n+1} k^{-n-1} .
$$

По определению полунормы $\nu_{V, n}($ см. (2.6)):

$$
\nu_{V, n}\left(g_{r}\right)=\left\|g_{r}\right\|_{\infty}+\sup _{k \in \mathbb{N}} k^{n}\left\|\left.g_{r}\right|_{G \backslash V^{k}}\right\|_{\infty} .
$$

При $1 \leqslant k \leqslant r$, пользуясь неравенством (2.23) и очевидным неравенством $\left\|\left.g_{r}\right|_{G \backslash V^{k}}\right\|_{\infty} \leqslant\left\|g_{r}\right\|_{\infty}$, получаем оценку

$$
k^{n}\left\|\left.g_{r}\right|_{G \backslash V^{k}}\right\|_{\infty} \leqslant r^{n}\left\|g_{r}\right\|_{\infty} \leqslant M_{n+1} r^{-1}, \quad 1 \leqslant k \leqslant r .
$$

При $k>r$, используя неравенство (2.24), получаем оценку

$$
k^{n}\left\|\left.g_{r}\right|_{G \backslash V^{k}}\right\|_{\infty} \leqslant M_{n+1} k^{-1} \leqslant M_{n+1} r^{-1}, \quad k>r .
$$

Из (2.26) и (2.27) вытекает, что неравенство

$$
k^{n}\left\|\left.g_{r}\right|_{G \backslash V^{k}}\right\|_{\infty} \leqslant M_{n+1} r^{-1}
$$

справедливо для любого $k \in \mathbb{N}$, поэтому из (2.25) и (2.23) следует, что

$$
\nu_{V, n}\left(g_{r}\right) \leqslant 2 M_{n+1} r^{-1} .
$$

Из (2.29) вытекает, что для любого $n \in \mathbb{N}$ последовательность $\nu_{V, n}\left(g_{r}\right)$ стремится к нулю при $r \rightarrow \infty$, откуда следует, что $f_{r} \rightarrow f$ в пространстве $\mathscr{A}(G)$.

Из свойств (2.18) и (2.19) вытекает, что функция $g_{r}=f\left(1-I_{r}\right)$ удовлетворяет условию

$$
g_{r}(x y)=g_{r}(x) \quad \forall x \in G, y \in U, \quad r \in \mathbb{N} .
$$

Пусть $H=A(\widehat{G}, U)$. Тогда $H$ является компактной открытой подгруппой группы $\widehat{G}$ и, рассуждая как в п. 2) доказательства предложения 2.5, можно доказать, что $\operatorname{supp}\left(\widehat{g_{r}}\right) \subseteq H$.

Из определения функции $g_{r}$ и из свойства $(\mathscr{A} 3)$ вытекает, что существует число $M>0$ такое, что для любого $r \in \mathbb{N}$ справедливо неравенство

$$
\left\|g_{r}\right\|_{1} \leqslant M r^{-1} \text {. }
$$

Из свойства (2.3) преобразования Фурье и из неравенства (2.31) следует, что

$$
\left\|\widehat{g_{r}}\right\|_{\infty} \leqslant\left\|g_{r}\right\|_{1} \leqslant M r^{-1}
$$

откуда вытекает, что $\widehat{g_{r}} \rightarrow 0$ в пространстве $L_{\mathrm{c}}^{\infty}(\widehat{G})$. Так как группа $\widehat{G}$ поэлементно компактная, то по предложению 2.3 топологические векторные пространства $\mathscr{A}(\widehat{G})$ и $L_{\mathrm{c}}^{\infty}(\widehat{G})$ совпадают, следовательно, $\widehat{g_{r}} \rightarrow 0$ и $\widehat{f}_{r} \rightarrow \widehat{f}$ в пространстве $\mathscr{A}(\widehat{G})$. Теорема 2.2 доказана. 


\section{§ 3. Доказательство теоремы 1.1}

Пусть $G$ - произвольная LCA-группа, $C(G)$ - множество непрерывных функций на $G, C_{\mathrm{c}}(G)$ - множество непрерывных функций на $G$ с компактным носителем. Множество $C(G)$ является локально выпуклым топологическим векторным пространством с топологией равномерной сходимости на компактных подмножествах в $G$.

Через $\mathscr{M}_{\mathrm{c}}(G)$ обозначим множество всех комплексных мер Радона с компактным носителем на локально компактном пространстве $G$. Через $\operatorname{supp}(\mu)$ будем обозначать носитель меры $\mu \in \mathscr{M}_{\mathrm{c}}(G)$. Множество $\mathscr{M}_{\mathrm{c}}(G)$ является комлексным линейным пространством. Пространство $\mathscr{M}_{\mathrm{c}}(G)$ можно отождествить с двойственным пространством к пространству $C(G)$, если определить двойственность по формуле

$$
\langle\mu, \varphi\rangle:=\int_{G} \varphi(x) d \mu(x),
$$

где $\varphi \in C(G), \mu \in \mathscr{M}_{\mathrm{c}}(G)$. Снабженное слабой топологией $\sigma\left(\mathscr{M}_{\mathrm{c}}(G), C(G)\right)$ пространство $\mathscr{M}_{\mathrm{c}}(G)$ является локально выпуклым пространством. Опишем топологию пространства $\mathscr{M}_{\mathrm{c}}(G)$ в более явном виде.

Для любой меры $\mu \in \mathscr{M}_{\mathrm{c}}(G)$ пусть $|\mu|-$ полная вариация меры $\mu$. Через $\tau_{0}(G)$ будем обозначать совокупность всех непустых открытых подмножеств группы $G$ с компактным замыканием. Если $U \in \tau_{0}(G)$, то через $\mathscr{M}_{\mathrm{c}}(U)$ обозначим множество всех мер, носители которых содержатся в множестве $[U]([U]-$ замыкание множества $U)$. Множество $\mathscr{M}_{\mathrm{c}}(U)$ является банаховым пространством с нормой $\|\mu\|:=|\mu|([U]), \mu \in \mathscr{M}_{\mathrm{c}}(U)$. Если $U, V \in \tau_{0}(G)$ и $V \subseteq U$, то $\mathscr{M}_{\mathrm{c}}(V) \subseteq \mathscr{M}_{\mathrm{c}}(U)$, причем вложение непрерывное. Топология в пространстве

$$
\mathscr{M}_{\mathrm{c}}(G)=\bigcup_{U \in \tau_{0}(G)} \mathscr{M}_{\mathrm{c}}(U)
$$

совпадает с топологией индуктивного предела банаховых пространств $\mathscr{M}_{\mathrm{c}}(U)$. Отметим, что если группа $G$ поэлементно компактна, то в силу предложения 2.2 можно считать, что $U$ пробегает множество всех компактных открытых подгрупп группы $G$.

Пространство $C_{\mathrm{c}}(G)$ вкладывается в пространство $\mathscr{M}_{\mathrm{c}}(U)$, если отождествить функцию $f \in C_{\mathrm{c}}(G)$ с мерой $f(x) d x$, где $d x$ - элемент меры Хаара на группе $G$.

Пусть $\mu_{1}, \mu_{2} \in \mathscr{M}_{\mathrm{c}}(G)$. Свертка мер $\mu_{1} * \mu_{2}$ определяется формулой

$$
\left\langle\mu_{1} * \mu_{2}, \varphi\right\rangle:=\int_{G} \int_{G} \varphi(x y) d \mu_{1}(x) d \mu_{2}(y),
$$

где $\varphi \in C(G)$ (см., например, [7] или [14]). Отметим, что $\operatorname{supp}\left(\mu_{1} * \mu_{2}\right) \subseteq$ $\operatorname{supp}\left(\mu_{1}\right) \operatorname{supp}\left(\mu_{2}\right)$. В частности, определена свертка $f * \mu$ для любой функции $f \in C_{\mathrm{c}}(G)$ и любой меры $\mu \in \mathscr{M}_{\mathrm{c}}(G)$. При этом $f * \mu \in C_{\mathrm{c}}(G)$ и свертка задается формулой

$$
(f * \mu)(x)=\int_{G} f\left(x y^{-1}\right) d \mu(y) .
$$


Множество $\mathscr{M}_{\mathrm{c}}(G)$ является коммутативной топологической алгеброй относительно свертки. Легко видеть, что если $\mu_{1} \in \mathscr{M}_{\mathrm{c}}\left(U_{1}\right)$ и $\mu_{2} \in \mathscr{M}_{\mathrm{c}}\left(U_{2}\right)$ $\left(U_{1}, U_{2} \in \tau_{0}(G)\right)$, то $\mu_{1} * \mu_{2} \in \mathscr{M}_{\mathrm{c}}\left(U_{1} U_{2}\right)$ и $\left\|\mu_{1} * \mu_{2}\right\| \leqslant\left\|\mu_{1}\right\|\left\|\mu_{2}\right\|$.

Для любого замкнутого линейного подпространства $\mathscr{H} \subseteq C(G)$ пусть $\mathscr{H}^{\perp}-$ его ортогональное дополнение (= аннулятор) в $\mathscr{M}_{\mathrm{c}}(G)$, т.е.

$$
\mathscr{H}^{\perp}:=\left\{\mu \in \mathscr{M}_{\mathrm{c}}(G):\langle\mu, f\rangle=0 \forall f \in \mathscr{H}\right\} .
$$

Предложение 3.1. Отображение $\mathscr{H} \mapsto \mathscr{H}^{\perp}$ устанавливает взаимно однозначное соответствие между множеством всех инвариантных подпространств в $C(G)$ и множеством всех замкнутых идеалов в топологической алгебре $\mathscr{M}_{\mathrm{c}}(G)$.

ДоказАтельство. Для любых $f \in C(G)$ и $\mu \in \mathscr{M}_{\mathrm{c}}(G)$ определим свертку $f * \mu$ по формуле (3.3). Тогда $f * \mu \in C(G)$ и если $\mathscr{H}$ - инвариантное подпространство в $C(G)$ и $f \in \mathscr{H}$, то $f * \mu \in \mathscr{H}$.

Пусть $\varepsilon_{a}$ - мера с носителем в точке $a \in G$, определяемая условием

$$
\int_{G} f(x) d \varepsilon_{a}(x)=f(a) \quad \forall f \in C(G),
$$

тогда

$$
\left(f * \varepsilon_{a}\right)(x)=f\left(x a^{-1}\right) \quad \forall f \in C(G) .
$$

С учетом (3.4) получаем, что замкнутое линейное подпространство $\mathscr{H} \subseteq C(G)$ является инвариантным подпространством тогда и только тогда, когда $\mathscr{H}$ инвариантно относительно сверток с мерами из $\mathscr{M}_{\mathrm{c}}(G)$, т.е. если $f \in \mathscr{H}, \mu \in$ $\mathscr{M}_{\mathrm{c}}(G)$, то $f * \mu \in \mathscr{H}$.

Для любой меры $\mu \in \mathscr{M}_{\mathrm{c}}(G)$ обозначим через $\tilde{\mu}$ меру из $\mathscr{M}_{\mathrm{c}}(G)$, определяемую формулой

$$
\langle\tilde{\mu}, \varphi\rangle:=\int_{G} \varphi\left(x^{-1}\right) d \mu(x), \quad \varphi \in C(G) .
$$

Легко видеть, что для любых $f \in C(G), \mu_{1}, \mu_{2} \in \mathscr{M}_{\mathrm{c}}(G)$ справедливо равенство

$$
\left\langle\mu_{1} * \mu_{2}, f\right\rangle=\left\langle\mu_{1}, f * \tilde{\mu}_{2}\right\rangle .
$$

Из теоремы Хана-Банаха следует, что отображение $\mathscr{H} \mapsto \mathscr{H}^{\perp}$ задает взаимно однозначное соответствие между замкнутыми линейными подпространствами пространств $C(G)$ и $\mathscr{M}_{\mathrm{c}}(G)$. Из (3.5) вытекает, что $\mathscr{H}$ инвариантно относительно сверток с мерами из $\mathscr{M}_{\mathrm{c}}(G)$ тогда и только тогда, когда $\mathscr{H}^{\perp}$ инвариантно относительно сверток с мерами из $\mathscr{M}_{\mathrm{c}}(G)$, т.е. $\mathscr{H}$ является инвариантным подпространством в $C(G)$ тогда и только тогда, когда $\mathscr{H}^{\perp}$ является замкнутым идеалом в $\mathscr{M}_{\mathrm{c}}(G)$.

Для любой меры $\mu \in \mathscr{M}_{\mathrm{c}}(G)$ ее преобразованием Фурье называется функция $\widehat{\mu}$ на группе $\widehat{G}$, определяемая по формуле

$$
\widehat{\mu}(\chi):=\int_{G} \overline{\chi(x)} d \mu(x), \quad \chi \in \widehat{G} .
$$


Так как $|\widehat{\mu}(\chi)| \leqslant|\mu|(\operatorname{supp} \mu)=\|\mu\|$, то $\widehat{\mu} \in L^{\infty}(\widehat{G})$. Легко видеть, что преобразование Фурье переводит свертку мер в произведение, т.е.

$$
\widehat{\mu_{1} * \mu_{2}}=\widehat{\mu}_{1} \widehat{\mu}_{2}, \quad \mu_{1}, \mu_{2} \in \mathscr{M}_{\mathrm{c}}(G) .
$$

ПРЕДЛОЖЕНИЕ 3.2. Если группа $G$ поэлементно компактная, то для любой меры $\mu \in \mathscr{M}_{\mathrm{c}}(G)$ функция $\widehat{\mu}$ будет равномерно локально постоянной функuцей на әрynne $\widehat{G}$.

ДокАзАтельство. Так как группа $G$ поэлементно компактная, то по предложению 2.2 существует компактная открытая подгруппа $H$ группы $G$ такая, что $\operatorname{supp}(\mu) \subseteq H$. Пусть $U=A(\widehat{G} ; H)$, тогда $U$ является компактной открытой подгруппой группы $\widehat{G}$. Покажем, что выполняется равенство

$$
\widehat{\mu}(\chi \xi)=\widehat{\mu}(\chi) \quad \forall \chi \in \widehat{G}, \xi \in U .
$$

Действительно, так как $\operatorname{supp}(\mu) \subseteq H$ и $\xi(x)=1$ при $x \in H$, то

$$
\widehat{\mu}(\chi \xi)=\int_{G} \overline{\chi(x) \xi(x)} d \mu(x)=\int_{H} \overline{\chi(x) \xi(x)} d \mu(x)=\int_{H} \overline{\chi(x)} d \mu(x)=\widehat{\mu}(\chi) .
$$

Из равенства (3.8) вытекает, что функция $\widehat{\mu}$ равномерно локально постоянная.

ПреДЛОЖЕНИЕ 3.3. Пусть $G$ - поэлементно компактная LCA-группа, $\varphi \in$ $\mathscr{S}(G)$. Тогда:

1) для любой меры $\mu \in \mathscr{M}_{\mathrm{c}}(G)$ функиия $\varphi * \mu$ принадлежст классу $\mathscr{S}(G)$;

2) отображение $\mu \mapsto \varphi * \mu$ является непрерывным отображением из ТВП $\mathscr{M}_{\mathrm{c}}(G)$ в $\mathrm{TB \Pi} \mathscr{S}(G)$.

ДоказАтельство. 1) Чтобы доказать, что $\varphi * \mu \in \mathscr{S}(G)$, нужно показать, что $\varphi * \mu \in \mathscr{A}(G)$ и $\widehat{\varphi * \mu} \in \mathscr{A}(\widehat{G})$.

Так как группа $G$ поэлементно компактная, то по следствию $2.1 \varphi$ является функцией с компактным носителем, т.е. $\varphi \in C_{\mathrm{c}}(G)$. Тогда $\varphi * \mu \in C_{\mathrm{c}}(G) \subseteq$ $L_{\mathrm{c}}^{\infty}(G)$, а так как $L_{\mathrm{c}}^{\infty}(G) \subseteq \mathscr{A}(G)$ (см. предложение 3.3), то $\varphi * \mu \in \mathscr{A}(G)$.

Так как $\varphi \in \mathscr{S}(G)$, то функция $\widehat{\varphi}$ принадлежит классу $\mathscr{A}(\widehat{G})$. Поскольку функция $\widehat{\mu}$ принадлежит классу $L^{\infty}(\widehat{G})$, то по свойству $(\mathscr{A} 3)$ произведение $\widehat{\varphi} \widehat{\mu}$ принадлежит классу $\mathscr{A}(\widehat{G})$. Так как $\widehat{\varphi * \mu}=\widehat{\varphi} \widehat{\mu}$, то $\widehat{\varphi * \mu} \in \mathscr{A}(\widehat{G})$.

2) Чтобы доказать, что отображение $\mu \mapsto \varphi * \mu$ является непрерывным отображением из ТВП $\mathscr{M}_{\mathrm{c}}(G)$ в ТВП $\mathscr{S}(G)$, достаточно показать, что это отображение непрерывно как отображение из пространства $\mathscr{M}_{\mathrm{c}}(G)$ в пространство $\mathscr{A}(G)$ и отображение $\mu \mapsto \widehat{\varphi * \mu}$ непрерывно как отображение из пространства $\mathscr{M}_{\mathrm{c}}(G)$ в пространство $\mathscr{A}(\widehat{G})$.

Пусть $H$ - компактная открытая подгруппа группы $G, \mu \in \mathscr{M}_{\mathrm{c}}(H)$ (т.е. $\operatorname{supp}(\mu) \subseteq H), U-$ компактная открытая подгруппа группы $G$ такая, что $\operatorname{supp}(\varphi) \subseteq U$. Пусть $W=U H$, тогда $W$ также является компактной открытой подгруппой группы $G$ и $\operatorname{supp}(\varphi * \mu) \subseteq W$.

Заметим, что для любого $x \in G$ справедлива оценка

$$
|(\varphi * \mu)|(x) \leqslant \int_{G}\left|\varphi\left(x y^{-1}\right)\right| d|\mu|(y) \leqslant\|\varphi\|_{\infty} \int_{H} d|\mu|(y)=\|f\|_{\infty}|\mu|(H)=\|\varphi\|_{\infty}\|\mu\|,
$$


откуда следует, что

$$
\|\varphi * \mu\|_{\infty} \leqslant\|\varphi\|_{\infty}\|\mu\| .
$$

Тем самым доказано, что для любой компактной открытой подгруппы $H \subseteq G$ существует компактная открытая подгруппа $W \subseteq G$ такая, что отображение $\mu \mapsto \varphi * \mu$ является линейным непрерывным отображением из банахова пространства $\mathscr{M}_{\mathrm{c}}(H)$ в банахово пространство $L_{\mathrm{c}}^{\infty}(W)$. Так как пространство $\mathscr{M}_{\mathrm{c}}(G)$ снабжается топологией индуктивного предела банаховых пространств $\mathscr{M}_{\mathrm{c}}(H), H \in \Lambda(G)$, а пространство $L_{\mathrm{c}}^{\infty}(G)$ снабжается топологией индуктивного предела банаховых пространств $L_{\mathrm{c}}^{\infty}(W), W \in \Lambda(G)$, то отображение $\mu \mapsto \varphi * \mu$ является непрерывным отображением из ТВП $\mathscr{M}_{\mathrm{c}}(G)$ в ТВП $L_{\mathrm{c}}^{\infty}(G)=\mathscr{A}(G)$.

Так как $\varphi \in \mathscr{S}(G)$, то $\widehat{\varphi} \in \mathscr{A}(\widehat{G})$ и, следовательно, $\widehat{\varphi} \in \mathscr{A}_{V}(\widehat{G})$ для некоторой симметричной компактной открытой окрестности единицы $V$ в группе $\widehat{G}$. По определению пространство $\mathscr{A}_{V}(\widehat{G})$ состоит из всех функций $f \in L^{\infty}(\widehat{G})$, удовлетворяющих условию $(2.5)$, в котором $G$ нужно заменить на $\widehat{G}$. Топология в локально выпуклом пространстве $\mathscr{A}_{V}(\widehat{G})$ задается системой полунорм

$$
\nu_{V, n}(f):=\|f\|_{\infty}+\sup _{k \in \mathbb{N}} k^{n}\left\|\left.f\right|_{\widehat{G} \backslash V^{k}}\right\|_{\infty}, \quad f \in \mathscr{A}_{V}(\widehat{G}), \quad n \in \mathbb{Z}_{+} .
$$

Так как $|\widehat{\mu}(\chi)| \leqslant\|\mu\|$ при $\mu \in \mathscr{M}_{\mathrm{c}}(G), \chi \in \widehat{G}$, то из (3.10), с учетом того, что $\widehat{\varphi * \mu}=\widehat{\varphi} \widehat{\mu}$, вытекает неравенство

$$
\nu_{V, n}(\widehat{\varphi * \mu})=\nu_{V, n}(\widehat{\varphi} \widehat{\mu}) \leqslant \nu_{V, n}(\varphi)\|\mu\| .
$$

Из (3.11) следует, что линейное отображение $\mu \mapsto \widehat{\varphi * \mu}$ является непрерывным отображением из ТВП $\mathscr{M}_{\mathrm{c}}(G)$ в ТВП $\mathscr{A}_{V}(\widehat{G})$, а следовательно, и непрерывным отображением из ТВП $\mathscr{M}_{\mathrm{c}}(G)$ в ТВП $\mathscr{A}(\widehat{G})$.

ЛЕмма 3.1. Для любого компактного подмножества $P \subseteq \widehat{G}$ и для любого $\varepsilon>0$ существует окрестность $U$ единицы в группе $G$ такая, что при всех $\chi \in P, x \in U$ справедливо неравенство $|\chi(x)-1|<\varepsilon$.

Доказательство см. в [7; п. 24.2].

Известно, что для любой окрестности $U$ единицы в LCA-группе $G$ существует функция $\varphi_{U} \in \mathscr{S}(G)$, удовлетворяющая условиям:

1) $\varphi(x) \geqslant 0$ для всех $x \in G$;

2) $\operatorname{supp}\left(\varphi_{U}\right) \subseteq U$;

3) $\int_{G} \varphi_{U}(x) d x=1$ (см. [12; предложение 2]).

Всюду далее $G$ - поэлементно компактная LCA-группа. Как и раньше, через $\mathfrak{V}=\mathfrak{V}(G)$ обозначим множество всех симметричных окрестностей единицы с компактным замыканием группы $G$. Если $U_{1}, U_{2} \in \mathfrak{V}$, то множество $U_{3}=U_{1} \cap U_{2}$ также принадлежит $\mathfrak{V}$; поэтому $\mathfrak{V}$ является направленным множеством относительно включения. Для каждого множества $U \in \mathfrak{V}(G)$ зафиксируем функцию $\varphi_{U} \in \mathscr{S}(G)$, удовлетворяющую приведенным выше условиям 1)-3). Направленность $\varphi_{U}, U \in \mathfrak{V}$, образует аппроксимативную единицу для сверточных алгебр $\mathscr{S}(G)$ и $\mathscr{M}_{c}(G)$ в следующем смысле. 
ПреДЛОЖенИЕ 3.4. 1) Если $f \in \mathscr{S}(G)$, то $\varphi_{U} * f \underset{\mathfrak{V}}{\longrightarrow} f$ в пространстве $\mathscr{S}(G)$.

2) Если $\mu \in \mathscr{M}_{\mathrm{c}}(G)$, то $\varphi_{U} * \mu \underset{\mathfrak{V}}{\longrightarrow} \mu$ в пространстве $\mathscr{M}_{\mathrm{c}}(G)$.

ДоказАтельство. Чтобы доказать, что $\varphi_{U} * f \underset{\mathfrak{V}}{\longrightarrow} f$ в пространстве $\mathscr{S}(G)$, достаточно доказать, что $\varphi_{U} * f \underset{\mathfrak{V}}{\longrightarrow} f$ в пространстве $\mathscr{A}(G)$ и $\widehat{\varphi_{U} * f} \underset{\mathfrak{V}}{\longrightarrow} \widehat{f}$ в пространстве $\mathscr{A}(\widehat{G})$.

Так как группа $G$ поэлементно компактная и $f \in \mathscr{S}(G)$, то из свойства $(\mathscr{S} 1)$ и из предложения 2.3 следует, что $f \in C_{\mathrm{c}}(G)$. Из того, что $f \in C_{\mathrm{c}}(G)$, легко получить, что $\varphi_{U} * f \underset{\mathfrak{V}}{\longrightarrow} f$ в пространстве $C_{\mathrm{c}}(G)$ и, в частности, в пространстве $L_{\mathrm{c}}^{\infty}(G)=\mathscr{A}(G)$.

Так как $f \in \mathscr{S}(G)$, то $\widehat{f} \in \mathscr{A}(\widehat{G})$ и, следовательно, $f \in \mathscr{A}_{V}(\widehat{G})$ для некоторого подмножества $V \in \mathfrak{V}(\widehat{G})$. Поскольку группа $\widehat{G}$ нульмерная, то по предложению 2.2 можно считать, что $V$ - компактное открытое подмножество в $\widehat{G}$.

Чтобы доказать, что $\widehat{\varphi_{U} * f}=\widehat{\varphi}_{U} \widehat{f} \underset{\mathfrak{V}}{\longrightarrow} \widehat{f}$ в локально выпуклом пространстве $\mathscr{A}_{V}(\widehat{G})$, нужно доказать, что для любых $\varepsilon>0$ и $n \in \mathbb{N}$ найдется окрестность $U_{0} \in \mathfrak{V}(G)$ такая, что для всякого $U \in \mathfrak{V}(G)$ из $U \subseteq U_{0}$ следует, что

$$
\nu_{V, n}\left(\widehat{\varphi}_{U} \widehat{f}-\widehat{f}\right) \leqslant \varepsilon,
$$

где $\nu_{V, n}$ - полунорма в $\mathscr{A}_{V}(\widehat{G})$, определенная в $(3.10)$.

Так как $\widehat{f} \in \mathscr{A}_{V}(\widehat{G})$, то существует число $M_{n+1}$ такое, что для любого $k \in \mathbb{N}$ справедливо неравенство

$$
\left\|\left.\widehat{f}\right|_{\widehat{G} \backslash V^{k}}\right\|_{\infty} \leqslant M_{n+1} k^{-n-1} .
$$

Отметим, что функция $\widehat{f}$ непрерывна на $\widehat{G}$ и

$$
|\widehat{f}(\chi)| \leqslant\|f\|_{1} \quad \forall \chi \in \widehat{G},
$$

где $\|f\|_{1}$ - норма $f$ в пространстве $L^{1}(G)$. В частности, из (3.14) и из свойства 3$)$ функции $\varphi_{U}$ следует, что

$$
\left|\widehat{\varphi}_{U}(\chi)\right| \leqslant 1 \quad \forall \chi \in \widehat{G} .
$$

Выберем число $k_{0} \in \mathbb{N}$ так, чтобы выполнялось неравенство

$$
M_{n+1} k_{0}^{-1} \leqslant \frac{\varepsilon}{4}
$$

Так как множество $V^{k_{0}}$ является компактным подмножеством в $\widehat{G}$, то по лемме 3.1 найдется окрестность $U_{0} \in \mathfrak{V}(G)$ такая, что

$$
|\chi(x)-1|<\frac{\varepsilon}{2 k_{0}^{n}\|f\|_{1}} \quad \forall \chi \in V^{k_{0}}, \quad x \in U_{0} .
$$

Пусть $U \subseteq U_{0}$. Оценим $\left\|\widehat{\varphi}_{U} \widehat{f}-\widehat{f}\right\|_{\infty}$. Рассмотрим два случая. 
а) Пусть $\chi \in V^{k_{0}}$. Так как $\operatorname{supp}\left(\varphi_{U}\right) \subseteq U_{0}$ и $\int_{U_{0}} \varphi_{U}(x) d x=1$, то

$$
\widehat{\varphi}_{U}(\chi)-1=\int_{U_{0}} \varphi_{U}(x)(\chi(x)-1) d x .
$$

Из (3.18), используя (3.17) и (3.14), получаем неравенство

$$
\left|\widehat{\varphi}_{U}(\chi) \widehat{f}(\chi)-\widehat{f}(\chi)\right|=\left|\widehat{\varphi}_{U}(\chi)-1\right||\widehat{f}(\chi)| \leqslant \frac{\varepsilon}{2 k_{0}^{n}\|f\|_{1}}\|f\|_{1}=\frac{\varepsilon}{2 k_{0}^{n}} .
$$

б) Пусть $\chi \notin V^{k_{0}}$, тогда из $(3.13),(3.15)$ и (3.16) следует неравенство

$$
\left|\widehat{\varphi}_{U}(\chi) \widehat{f}(\chi)-\widehat{f}(\chi) \leqslant\left\|\left.\left(\widehat{\varphi}_{U}(\chi)-1\right) \widehat{f}\right|_{\widehat{G} \backslash V^{k_{0}}}\right\|_{\infty} \leqslant 2 M_{n+1} k_{0}^{-n-1} \leqslant \frac{\varepsilon}{2 k_{0}^{n}} .\right.
$$

Из (3.19) и (3.20) вытекает, что

$$
\left\|\widehat{\varphi}_{U} \widehat{f}-\widehat{f}\right\|_{\infty} \leqslant \frac{\varepsilon}{2 k_{0}^{n}}
$$

Оценим числа $k^{n}\left\|\left.\left(\widehat{\varphi}_{U} \widehat{f}-\widehat{f}\right)\right|_{\widehat{G} \backslash V^{k}}\right\|_{\infty}, k \in \mathbb{N}$. При $k \leqslant k_{0}$ воспользуемся неравенством (3.21), тогда

$$
k^{n}\left\|\left.\left(\widehat{\varphi}_{U} \widehat{f}-\widehat{f}\right)\right|_{\widehat{G} \backslash V^{k}}\right\|_{\infty} \leqslant k_{0}^{n}\left\|\widehat{\varphi}_{U} \widehat{f}-\widehat{f}\right\|_{\infty} \leqslant \frac{\varepsilon}{2} .
$$

При $k>k_{0}$ воспользуемся неравенствами $(3.13),(3.15)$ и (3.16), тогда

$$
k^{n}\left\|\left.\left(\widehat{\varphi}_{U} \widehat{f}-\widehat{f}\right)\right|_{\widehat{G} \backslash V^{k}}\right\|_{\infty} \leqslant 2 k^{n}\left\|\left.\widehat{f}\right|_{\widehat{G} \backslash V^{k}}\right\|_{\infty} \leqslant 2 M_{n+1} k^{-1} \leqslant 2 M_{n+1} k_{0}^{-1} \leqslant \frac{\varepsilon}{2} .
$$

Из (3.22) и (3.23) вытекает, что

$$
k^{n}\left\|\left.\left(\widehat{\varphi}_{U} \widehat{f}-\widehat{f}\right)\right|_{\widehat{G} \backslash V^{k}}\right\|_{\infty} \leqslant \frac{\varepsilon}{2} \quad \forall k \in \mathbb{N},
$$

откуда, с учетом (3.21), следует неравенство

$$
\nu_{V, n}\left(\widehat{\varphi}_{U} \widehat{f}-\widehat{f}\right)=\left\|\widehat{\varphi}_{U} \widehat{f}-\widehat{f}\right\|_{\infty}+\sup _{k \in \mathbb{N}} k^{n}\left\|\left.\left(\widehat{\varphi}_{U} \widehat{f}-\widehat{f}\right)\right|_{\widehat{G} \backslash V^{k}}\right\|_{\infty} \leqslant \frac{\varepsilon}{2}+\frac{\varepsilon}{2}=\varepsilon
$$

что доказывает (3.12) и завершает доказательство пункта 1).

2) Так как топология пространства $\mathscr{M}_{\mathrm{c}}(G)$ совпадает со слабой топологией $\sigma\left(\mathscr{M}_{\mathrm{c}}(G), C(G)\right)$, то для доказательства того, что $\varphi_{U} * \mu \underset{\mathfrak{V}}{\longrightarrow} \mu$ в пространстве $\mathscr{M}_{\mathrm{c}}(G)$, достаточно доказать, что $\left\langle\varphi_{U} * \mu, g\right\rangle \underset{\mathfrak{V}}{\longrightarrow}\langle\mu, g\rangle$ для любой функции $g \in$ $C(G)$.

Заметим, что

$$
\left\langle\varphi_{U} * \mu, g\right\rangle=\left\langle\mu, g * \widetilde{\varphi}_{U}\right\rangle,
$$

где $\widetilde{\varphi}_{U}(x):=\varphi_{U}\left(x^{-1}\right)$. Легко видеть, что $g * \widetilde{\varphi}_{U} \underset{\mathfrak{V}}{\longrightarrow} g$ в пространстве $C(G)$, откуда, используя равенство (3.25), получаем, что

$$
\left\langle\varphi_{U} * \mu, g\right\rangle=\left\langle\mu, g * \widetilde{\varphi}_{U}\right\rangle \underset{\mathfrak{V}}{\longrightarrow}\langle\mu, g\rangle .
$$

Предложение 3.4 доказано. 
Для любой топологической алгебры $\mathscr{A}$ через $s(\mathscr{A})$ будем обозначать множество всех замкнутых идеалов алгебры $\mathscr{A}$. В частности, возникают множества $s\left(\mathscr{M}_{\mathrm{c}}(G)\right)$ и $s\left(\mathscr{S}_{\text {conv }}(G)\right)$. Используя отождествление функции $f \in \mathscr{S}(G)$ с мерой $f(x) d x$, будем считать, что $\mathscr{S}(G) \subseteq \mathscr{M}_{\mathrm{c}}(G)$. Определим отображения $\rho: s\left(\mathscr{M}_{\mathrm{c}}(G)\right) \mapsto s\left(\mathscr{S}_{\text {conv }}(G)\right)$ и $\tilde{\rho}: s\left(\mathscr{S}_{\text {conv }}(G)\right) \mapsto s\left(\mathscr{M}_{\mathrm{c}}(G)\right)$ формулами:

$$
\begin{array}{rlrl}
\rho(\mathscr{H}) & :=\mathscr{H} \cap \mathscr{S}(G), & & \mathscr{H} \in s\left(\mathscr{M}_{\mathrm{c}}(G)\right), \\
\tilde{\rho}\left(\mathscr{H}_{0}\right):=\left[\mathscr{H}_{0}\right], & & \mathscr{H}_{0} \in s\left(\mathscr{S}_{\text {conv }}(G)\right),
\end{array}
$$

где $\left[\mathscr{H}_{0}\right]$ - замыкание множества $\mathscr{H}_{0}$ в пространстве $\mathscr{M}_{\mathrm{c}}(G)$.

ПреДЛОЖениЕ 3.5. Отображение $\rho$ является биекцией множества $s\left(\mathscr{M}_{\mathrm{c}}(G)\right)$ на множество $s\left(\mathscr{S}_{\mathrm{conv}}(G)\right)$. При этом обратное отображение $\rho^{-1}$ cовпадает с $\tilde{\rho}$.

ДокАЗАТЕльство. Пусть $\mathscr{H}$ - произвольный замкнутый идеал в алгебре $\mathscr{M}_{\mathrm{c}}(G), \mathscr{H}_{0}=\mathscr{H} \cap \mathscr{S}(G)$. Если $\mu \in \mathscr{H}$, а $\varphi_{U}$ - определенные выше функции, $U \in \mathfrak{V}(G))$, то из предложения 3.3 следует, что $\varphi_{U} * \mu \in \mathscr{H} \cap \mathscr{S}(G)=\mathscr{H}_{0}$. По предложению $3.4 \varphi_{U} * \mu \underset{\mathfrak{V}}{\longrightarrow} \mu$ в пространстве $\mathscr{M}_{\mathrm{c}}(G)$, поэтому $\mu \in\left[\mathscr{H}_{0}\right]$. Следовательно, $\left[\mathscr{H}_{0}\right]=\mathscr{H}$, т.е. $\tilde{\rho} \circ \rho=\operatorname{Id}_{s\left(\mathscr{M}_{\mathrm{c}}(G)\right)}\left(\operatorname{Id}_{A}-\right.$ тождественное отображение множества $A$ в себя).

Теперь пусть $\mathscr{H}_{0}$ - произвольный замкнутый идеал в $\mathscr{S}_{\text {conv }}(G), \mathscr{H}=\left[\mathscr{H}_{0}\right]$. Если $f \in \mathscr{H} \cap \mathscr{S}(G)$, то найдется направленность $\left\{h_{\omega}\right\}_{\omega \in \Omega}(\Omega$ - некоторое направленное множество) такая, что $h_{\omega} \in \mathscr{H}_{0}$ и $h_{\omega} \underset{\Omega}{\longrightarrow} f$ в пространстве $\mathscr{M}_{\mathrm{c}}(G)$. Из предложения 3.3 вытекает, что отображение $\mu \mapsto \varphi_{P} * \mu$ является непрерывным отображением из $\mathscr{M}_{\mathrm{c}}(G)$ в $\mathscr{S}(G)$, поэтому из того, что $h_{\omega} \underset{\Omega}{\longrightarrow} f$ в $\mathscr{M}_{\mathrm{c}}(G)$, следует, что $\varphi_{P} * h_{\omega} \underset{\Omega}{\longrightarrow} \varphi_{P} * f$ в пространстве $\mathscr{S}(G)$. Так как $\varphi_{P} * h_{\omega} \in \mathscr{H}_{0}$ и $\mathscr{H}_{0}$ замкнуто в $\mathscr{S}(G)$, то $\varphi_{P} * f \in \mathscr{H}_{0}$. Так как $f \in \mathscr{S}(G)$, то $\varphi_{P} * f \underset{\mathfrak{V}}{\longrightarrow} f$ в пространстве $\mathscr{S}(G)$ (предложение $3.4,1)$ ), следовательно, $f \in \mathscr{H}_{0}$. Тем самым получено, что $\mathscr{H} \cap \mathscr{S}(G)=\mathscr{H}_{0}$, т.е. $\rho \circ \tilde{\rho}=\operatorname{Id}_{s\left(\mathscr{S}_{\text {conv }}(G)\right)}$.

Пусть $\Phi: f(x) \mapsto \widehat{f}(\chi)$ - преобразование Фурье. Так как $\Phi$ является изоморфизмом ТВП $\mathscr{S}(G)$ на ТВП $\mathscr{S}(\widehat{G})$ (свойство $(\mathscr{S} 2))$ и $\Phi$ переводит свертку функций в произведение, то $\Phi$ является изоморфизмом топологических алгебр $\mathscr{S}_{\text {conv }}(G)$ и $\mathscr{S}_{\text {mult }}(\widehat{G})$.

ДокАЗАТЕЛЬСТво теОРЕмы 1.1. Пусть $\mathscr{H}$ - инвариантное подпространство в $C(G), \mathscr{H}^{\perp}-$ его ортогональное дополнение в пространстве $\mathscr{M}_{\mathrm{c}}(G), \mathscr{I}=$ $\mathscr{H}^{\perp} \cap \mathscr{S}(G), \widehat{\mathscr{I}}=\Phi(\mathscr{I})$. Тогда $\mathscr{I}$ является замкнутым идеалом в топологической алгебре $\mathscr{S}_{\text {conv }}(G)$, а $\widehat{\mathscr{I}}$ является замкнутым идеалом в топологической алгебре $\mathscr{S}_{\text {mult }}(\widehat{G})$. Будем говорить, что идеал $\widehat{\mathscr{I}}$ соответствует инвариантному подпространству $\mathscr{H}$.

Пусть $\chi \in \widehat{G}$. Докажем, что $\chi \in \mathscr{H}$ тогда и только тогда, когда точка $\chi$ является общим нулем всех функций из идеала $\widehat{\mathscr{I}}$. Действительно, если $\chi \in \mathscr{H}$, то $\langle\mu, \chi\rangle=0$ для любого $\mu \in \mathscr{H}^{\perp}$, откуда вытекает, что $\langle f, \chi\rangle=0$ для любой 
функции $f \in \mathscr{I}$. Так как $\langle f, \chi\rangle=\widehat{f}(\chi)$, то $\widehat{f}(\chi)=0$ для любой функции $\widehat{f} \in \widehat{\mathscr{I}}$, т.е. $\chi$ является общим нулем для всех функций из $\widehat{\mathscr{I}}$.

Обратно, если $\widehat{f}(\chi)=0$ для всех $\widehat{f} \in \widehat{\mathscr{I}}$, то $\langle\widehat{f}, \chi\rangle=0$ для любой функции $f \in \mathscr{I}$. Так как замыкание идеала $\mathscr{I}$ в $\mathscr{M}_{\mathrm{c}}(G)$ совпадает с $\mathscr{H}^{\perp}$ (см. предложение 3.5), то $\langle\mu, \chi\rangle=0$ для любой меры $\mu \in \mathscr{H}^{\perp}$, откуда вытекает, что $\chi \in \mathscr{H}$.

Таким образом, спектр $\sigma(\mathscr{H})$ инвариантного подпространства $\mathscr{H}$ совпадает с нулевым множеством $N\left(\widehat{\mathscr{I})}\right.$ соответствующего ему идеала $\widehat{\mathscr{I}} \subseteq \mathscr{S}_{\text {mult }}(\widehat{G})$.

Пусть $\mathscr{H}$ - инвариантное подпространство в $C(G)$. Обозначим через $\mathscr{H}_{1}$ подпространство в $C(G)$, которое является замыканием линейной оболочки всех характеров $\chi$, принадлежащих $\mathscr{H}$. Тогда $\mathscr{H}_{1}$ тоже будет инвариантным подпространством, $\mathscr{H}_{1} \subseteq \mathscr{H}$ и $\sigma(\mathscr{H})=\sigma\left(\mathscr{H}_{1}\right)$. Пусть $\mathscr{I}_{1}=\mathscr{H}_{1}^{\perp} \cap \mathscr{S}(G)$, $\widehat{\mathscr{I}_{1}}=\Phi\left(\mathscr{I}_{1}\right)$. Так как $N\left(\widehat{\mathscr{I})}=N\left(\widehat{\mathscr{I}_{1}}\right)\right.$, то из теоремы 2.2 следует, что $\widehat{\mathscr{I}}=\widehat{\mathscr{I}_{1}}$, а тогда из предложений 3.1 и 3.5 вытекает, что $\mathscr{H}=\mathscr{H}_{1}$, т.е. $\mathscr{H}$ допускает спектральный синтез.

Заметим, что отображение $\theta: \mathscr{H} \mapsto \widehat{\mathscr{I}}$, которое сопоставляет инвариантному подпространству $\mathscr{H}$ соответствующий ему идеал $\widehat{\mathscr{I}}$, является биекцией множества всех инвариантных подпространств пространства $C(G)$ на множество всех замкнутых идеалов топологической алгебры $\mathscr{S}_{\text {mult }}(\widehat{G})$. Это вытекает из того, что $\theta$ является композицией трех биективных отображений: $\theta=\Phi \circ \rho \circ \alpha$, где $\alpha: \mathscr{H} \mapsto \mathscr{H}^{\perp}-$ отображение из множества инвариантных подпространств пространства $C(G)$ в множество замкнутых идеалов топологической алгебры $\mathscr{M}_{\mathrm{c}}(G)$ (см. предложение 3.1), $\rho: \mathscr{H}^{\perp} \mapsto \mathscr{H}^{\perp} \cap \mathscr{S}(G)$ - отображение из множества замкнутых идеалов алгебры $\mathscr{M}_{\mathrm{c}}(G)$ в множество замкнутых идеалов топологической алгебры $\mathscr{S}_{\text {conv }}(G)$ (см. предложение 3.5$)$ и $\Phi: \mathscr{I} \mapsto \widehat{\mathscr{I}}$ - отображение из множества замкнутых идеалов алгебры $\mathscr{S}_{\text {conv }}(G)$ в множество замкнутых идеалов топологической алгебры $\mathscr{S}_{\text {mult }}(\widehat{G})$. Поэтому всевозможные спектры инвариантных подпространств пространства $C(G)$ совпадают со всевозможными нулевыми множествами замкнутых идеалов алгебры $\mathscr{S}_{\text {mult }}(\widehat{G})$, т.е. со всевозможными замкнутыми подмножествами $\sigma \subseteq \widehat{G}$ (см. теорему 2.2).

Из теоремы 1.1 вытекает, что если группа $G$ поэлементно компактна, то в пространстве $C(G)$ справедлив строгий спектральный синтез. Оказывается, что условие поэлементной компактности группы $G$ является также и необходимым условием для справедливости строгого спектрального синтеза в $C(G)$. Более точно, справедливо следующее предложение.

ПрЕДЛОжЕнИЕ 3.6. Если LCA-группа $G$ не является поэлементно компактной, то в пространстве $C(G)$ существует двумерное инвариантное подпространство, не допускающее строгий спектральныи синтез.

Для доказательства предложения 3.6 воспользуемся следующим результатом. 
ПреДЛОжЕНИЕ 3.7. Пустъ $G$ - LCA-группа, $B$ - замкнутая подгруппа ее компактных элементов. Для того чтобы для элементов $x, y \in G$ существовал непрерывный вещественный характер $f$ группы $G$ (т.е. непрерывный гомоморфизм из группь $G$ в аддитивную группу $\mathbb{R}$ вещественных чисел), удовлетворяющий условию $f(x) \neq f(y)$, необходимо и достаточно, чтобы $x y^{-1} \notin B$.

Доказательство см. [7; гл. 6, теорема 24.34].

ДоКАЗАТЕЛЬСтво ПРЕДЛОЖЕНИЯ 3.6. Пусть группа $G$ не является поэлементно компактной, и пусть $x_{0}$ - некомпактный элемент группы $G$. Воспользовавшись предложением 3.7 для случая, когда $x=x_{0}, y=e$, получим, что существует непрерывная функция $f: G \mapsto \mathbb{R}$, удовлетворяющая условиям:

1) $f(x y)=f(x)+f(y)$ для всех $x, y \in G$;

2) $f\left(x_{0}\right) \neq 0$.

Пусть подпространство $\mathscr{H} \subseteq C(G)$ состоит из всех функций вида $F(x)=$ $\lambda_{1}+\lambda_{2} f(x), \lambda_{1}, \lambda_{2} \in \mathbb{C}$. Тогда $\mathscr{H}$ является 2-мерным инвариантным подпространством в $C(G)$ и легко видеть, что единственной экспоненциальной функцией, содержащейся в $\mathscr{H}$, является характер $\chi_{0}(x) \equiv 1$. Следовательно, $\mathscr{H}$ не допускает строгий спектральный синтез.

\section{Список литературы}

[1] L. Schwartz, "Théorie générale des fonctions moyenne-périodiques", Ann. of Math. (2), 48:4 (1947), 857-929.

[2] Д. И. Гуревич, "Контрпримеры к проблеме Л. Шварца", Функи. анализ и его прил., 9:2 (1975), 29-35; англ. пер.: D. I. Gurevich, "Counterexamples to a problem of L. Schwartz", Funct. Anal. Appl., 9:2 (1975), 116-120.

[3] L. Schwartz, "Analyse et synthèse harmoniques dans les espaces de distributions", Canadian J. Math., 3 (1951), 503-512.

[4] С. С. Платонов, "О спектральном синтезе на нульмерных абелевых группах", $M a-$ тем. сб., 204:9 (2013), 99-114; англ. пер.: S. S. Platonov, "On spectral synthesis on zero-dimensional Abelian groups", Sb. Math., 204:9 (2013), 1332-1346.

[5] L. Székelyhidi, Discrete spectral synthesis and its applications, Springer Monogr. Math., Springer, Dordrecht, 2006, xvi+117 pp.

[6] M. Laczkovich, L. Székelyhidi, "Spectral synthesis on discrete Abelian groups", Math. Proc. Cambridge Philos. Soc., 143:1 (2007), 103-120.

[7] Э. Хьюитт, К.А. Росс, Абстрактный гармонический анализ, т. I, Наука, М., 1975, 654 с.; пер. с англ.: E. Hewitt, K. A. Ross, Abstract harmonic analysis, v. I: Structure of topological groups. Integration theory, group representations, Grundlehren Math. Wiss., 115, Academic Press, Inc., Publishers, New York; Springer-Verlag, Berlin-Göttingen-Heidelberg, 1963, viii+519 pp.

[8] Л. С. Понтрягин, Непрерывные группы, 3-е изд., Наука, М., 1973, 519 с.; англ. пер. 1-го изд.: L. S. Pontrjagin, Topological groups, Princeton Math. Ser., 2, Princeton Univ. Press, Princeton, 1939, ix+299 pp.

[9] С. А. Моррис, Двойственность Понтрягина и строение локально компактных абелевых груnп, Мир, М., 1980, 104 с.; пер. с англ.: S. A. Morris, Pontryagin duality and the structure of locally compact abelian groups, London Math. Soc. Lecture Note Ser., 29, Cambridge Univ. Press, Cambridge-New York-Melbourne, 1977, viii+128 pp. 
[10] L. Székelyhidi, "Spectral synthesis problems on locally compact groups", Monatsh. Math., 161:2 (2010), 223-232.

[11] J. Braconnier, "Sur les groupes topologiques localement compacts", J. Math. Pures Appl. (9), 27 (1948), 1-85.

[12] F. Bruhat, "Distributions sur un groupe localement compact et applications à l'étude des représentations des groupes p-adiques", Bull. Soc. Math. France, 89 (1961), 43-75.

[13] M. S. Osborne, "On the Schwartz-Bruhat space and Paley-Wiener theorem for locally compact Abelian groups", J. Functional Analysis, 19:1 (1975), 40-49.

[14] Р. Эдвардс, Функиионалъный анализ. Теория и приложения, Мир, М., 1969, 1071 с.; пер. с англ.: R. E. Edwards, Functional analysis. Theory and applications, Holt, Rinehart and Winston, New York-Toronto-London, 1965, xiii+781 pp.

\section{Сергей Сергеевич Платонов}

Поступила в редакцию

(Sergey S. Platonov)

01.09 .2014

Петрозаводский государственный университет

E-mail: platonov@psu.karelia.ru 